\title{
Deflagration Regimes of Laminar Flames Modeled after the Ozone Decomposition Flame
}

\section{B. ROGG A. LIÑÁN F. A. WILLIAMS}

\section{INTRODUCTION}

Since the reaction mechanism of the ozone decomposition flame is the simplest real one having all requisite features of a truly detailed mechanism, this flame has often attracted attention of researchers involved in theoretical studies of flame propagation. In particular, frequently it has been used as a vehicle for testing computational methods [1-5], which are powerful tools for analyzing flame structure. These methods provide global information like numerical values of burning velocities and profiles of variables of interest, but often they are not carried to the point of localizing and identifying various interesting phenomena occurring in a flame. As an alternative and potentially less laborious approach to identifying these phenomena, in the present work activation-energy asymptotics are employed for a detailed mechanism modeled after that of ozone decomposition. 
Recent studies of the ozone decomposition flame by activation-energy asymptotics [6, 7] are extended here to map possible additional regimes of combustion. Besides identifying different flame structures, further comparisons to numerical results for the ozone flame will be made.

\section{REACTION SCHEME}

The reaction scheme to be considered here can be written as

$$
\begin{array}{lc}
\mathrm{R}+\mathrm{M} \rightarrow \mathrm{P}+\mathrm{O}+\mathrm{M} & \left(Q_{1}=-D\right), \\
\mathrm{R}+\mathrm{O} \rightarrow 2 \mathrm{P} \quad\left(Q_{2}=2 Q+D\right), \\
2 \mathrm{O}+\mathrm{M} \rightarrow \mathrm{P}+\mathrm{M} \quad\left(Q_{3}=2 Q+2 D\right), \\
\mathrm{P}+\mathrm{O}+\mathrm{M} \rightarrow \mathrm{R}+\mathrm{M} \quad\left(Q_{4}=D\right),
\end{array}
$$

where $Q_{i}$ denotes the energy released in reaction $i$. In the first reaction the reactant $R$ dissociates into a product $\mathrm{P}$ and an active intermediary $\mathrm{O}$ through collision with any species $M$, which may be $R, P$, or $\mathrm{O}$. This first step will be called the generation step since it generates the important intermediary $\mathrm{O}$; it is endothermic, with a heat of dissociation $D$, and its rate constant has an activation energy $E$ $(\geq D$ ) which will be used in defining the expansion parameter for the treatment by activation-energy asymptotics. In the second reaction the active species $O$ collides with the reactant to produce two product molecules and to release the energy $2 Q+$ $D$, where $Q$ denotes the heat release associated with the overall reaction $R \rightarrow(3 / 2) P$, i.e., the heat of decomposition per mole for $\mathrm{R}$. Since the heat released in the second step often is that which is relevant to the burning velocity, this step will be called the exothermic step. In addition to these two-body steps, there are two three-body reactions (with the third body $M$ ) in which the active intermediary can recombine exothermicly, viz., the recombination to product represented by the third reaction and the recombination to reactant represented by the fourth, the reverse of the first. The product is assumed to be so stable that the reverse reactions of the second and third steps are entirely negligible under all conditions. In addi- tion, the last three steps are assigned zero activation energies, a generally valid selection for the recombination steps and often a reasonable approximation for the exothermic step, whose activation energy always is appreciably below that of the generation step.

In the application to the ozone decomposition flame $\mathrm{R}$ is the ozone molecule $\mathrm{O}_{3}, \mathrm{P}$ is the oxygen molecule $\mathrm{O}_{2}$ and $\mathrm{O}$ represents the oxygen atom. For this flame it has been shown [6] that in the primary reaction zone the reverse reactions of the second and third steps are entirely negligible for all pressures and initial $\mathrm{O}_{3}$ concentrations of interest, at least if the final temperature is below $5000 \mathrm{~K}$. For final temperatures above $2000 \mathrm{~K}$, at pressures below atmospheric, the equilibrium $\mathrm{O}$ atom concentration in the final mixture begins to become significant through the reverse of (3), but this does not influence the burning velocity. Thus for the main properties of interest in the ozone flame, the adopted reaction scheme provides a good description, even though it always predicts pure species $P$ in the burned gas. The ozone flame has, approximately, $Q=35 \mathrm{kcal} / \mathrm{mol}, D=25$ $\mathrm{kcal} / \mathrm{mol}$, and $E=22 \mathrm{kcal} / \mathrm{mol}$, according to current data. ${ }^{1}$ The rate parameters for the exothermic step are the least certain [5]; quoted activation energies range from 4 to $6 \mathrm{kcal} / \mathrm{mol}[1-5,8]$, which values are small enough to be approximated as zero (but not with high accuracy). An alternative to the basic problem described above is to assign the exothermic step an activation energy of $b E$ and to treat the ratio $b(0.18-0.27)$ as a parameter of order unity. Although most of the analysis to be pursued will address the basic problem $(b=0)$, some results will be given for $b$ $=0.206$ (as in [6]). For the ozone flame it has been shown [6] that there are important regimes of flame propagation in which the recombination steps are unimportant in determining the burning velocity, so that the generation and exothermic steps alone form a relevant submechanism. The chaperon efficiencies of $\mathrm{O}_{3}, \mathrm{O}_{2}$, and $\mathrm{O}$ as $\mathrm{M}$ are

\footnotetext{
Here the result that $E<D$ is a consequence of including the temperature dependence of the prefactor in the definition of an effective value of $E$ for convenience in analysis.
} 
known to differ by more than a factor of 2 for reactions (1) and (4) and of 3 (because of high efficiency of the $O$ atom) for (3) [6], but the concentrations usually are so different that with reasonable accuracy these differences can be ignored by judiciously selecting rates appropriate to the dominant third body (usually P). Therefore no distinction in efficiencies for different $M$ will be made here; the concentration of $\mathrm{M}$ will be taken to be the total number of moles per unit volume.

\section{FORMULATION}

Attention is focused on steady, planar flames in ideal gases with negligible radiant energy transfer, body forces, and Soret and Dufour effects. The pressure is taken to be constant and viscous dissipation negligible because of the low Mach number. It is evident for the ozone flame and implied in general by (1) and (2) that the ratios of molecular weight of $\mathrm{R} / \mathrm{P} / \mathrm{O}$ are $3 / 2 / 1$. Therefore the subscripts 1,2 , and 3 will be taken to refer to species $\mathrm{O}, \mathrm{P}$, and $\mathrm{R}$, respectively. The conservation equations employed will be those for energy and for species 1 and 3, with concentrations of species 2 to be obtained from overall mass conservation. Specific heats of all species will be taken to be a constant $c_{\mathrm{p}}$, and Fick's law will be applied with Lewis numbers $L_{i}$ constant for $i=1$, 3. In general appreciable errors can arise when these approximations are employed in activationenergy asymptotics [9], but for the ozone flame there are many conditions under which, through cancellation of errors, they provide excellent accuracy if the properties $c_{\mathrm{p}}, L_{i}$, and $\lambda$ (thermal conductivity) are evaluated at the flame temperature and composition [6]. In addition, to the extent to which tests have been performed it has been found that differences between results obtained by one-term and two-term expansions in the activation-energy parameter are fortuitously small for the ozone flame [6]; all expansions are carried only to leading order herein. These decisions are motivated mainly by the intent of exhibiting the key results as simply as possible; any unusual accuracy for ozone is a bonus.

Conservation for thermal energy, $O$, and $R$ can be written in nondimensional form as

$$
\begin{aligned}
\frac{d \theta}{d x}= & \frac{d^{2} \theta}{d x^{2}}+\frac{1}{Y_{3 \mathrm{u}}} \Lambda\left[-\left(\frac{1-\mu}{\mu}\right) \kappa_{1} Y_{3} Y_{4}\right. \\
& +\left(\frac{1+\mu}{\mu}\right) \kappa_{2} Y_{1} Y_{3}+\left(\frac{1}{\mu}\right) \kappa_{3} Y_{1}{ }^{2} \\
& \left.+\left(\frac{1-\mu}{\mu}\right) \kappa_{4} Y_{1} Y_{2}\right],
\end{aligned}
$$

$$
\begin{aligned}
\frac{d Y_{1}}{d x}= & \frac{1}{L_{1}} \frac{d^{2} Y_{1}}{d x^{2}} \\
& +\frac{1}{3} \Lambda\left(\kappa_{1} Y_{3} Y_{4}-\kappa_{2} Y_{1} Y_{3}\right. \\
- & \left.\kappa_{3} Y_{1}{ }^{2}-\kappa_{4} Y_{1} Y_{2}\right),
\end{aligned}
$$

and

$\frac{d Y_{3}}{d x}=\frac{1}{L_{3}} \frac{d^{2} Y_{3}}{d x^{2}}-\Lambda\left(\kappa_{1} Y_{3} Y_{4}+\kappa_{2} Y_{1} Y_{3}-\kappa_{4} Y_{1} Y_{2}\right)$,

where $x$ is the nondimensional distance, $\theta$ the nondimensional temperature, and $Y_{i}$ the mass fraction of species $i$. Specifically, in terms of the velocity $v$, density $\rho$, and physical distance $x^{\prime}, x$ $\equiv \int_{0}^{x^{\prime}}\left(\rho v c_{\mathrm{p}} / \lambda\right) d x^{\prime}$, and in terms of the temperature $T$ and molecular weight $W$ of the intermediate $\mathrm{O}$,

$\theta \equiv 3 W c_{\mathrm{p}}\left(T-T_{\mathrm{u}}\right) /\left(Q Y_{3 \mathrm{u}}\right)$,

where the subscript $\mathrm{u}$ identifies conditions in the fresh mixture at $x=-\infty$. Here $Y_{4}$ denotes the ratio of $W$ to the average molecular weight of the mixture and is given by

$Y_{4}=Y_{1}+\frac{1}{2} Y_{2}+\frac{1}{3} Y_{3}$.

From overall mass conservation

$Y_{2}=1-Y_{1}-Y_{3}$.

The energetic parameter $\mu \equiv(1+D / Q)^{-1}$ measures the ratio of the heat of dissociation to the heat of reaction, so that

$D / Q=(1-\mu) / \mu$, 
which goes from zero at $\mu=1$ to infinity at $\mu=$ 0 .

With the specific reaction-rate constant for (1) written as

$k_{1}=A e^{-E / R^{\circ} T}$

(where $A$ is the preexponential factor and $R^{\circ}$ the universal gas constant), the burning-rate eigenvalue has been defined as

$\Lambda=\lambda_{\mathrm{f}} A_{\mathrm{f}} e^{-E / R^{\circ} T_{\mathrm{f}} /\left(W c_{\mathrm{p}} v_{\mathrm{f}}^{2}\right),}$

where the subscript $f$ identifies conditions at the downstream end of a reaction zone that controls the deflagration velocity. Here $T_{\mathrm{f}}$ may differ from the final equilibrium temperature $T_{\mathrm{e}}=T_{\mathrm{u}}+$ $Y_{3 \mathrm{u}} Q /\left(3 W c_{\mathrm{p}}\right)$, corresponding to $\theta=1$, e.g., since (3) and (4) may cause further increases in temperature downstream from the primary reaction zone. The rate-constant ratios $\kappa_{i}$ essentially measure rates with respects to $k_{1 f}$. Specifically,

$\kappa_{1}=\left(\rho / \rho_{\mathrm{f}}\right)^{2}\left(\lambda / \lambda_{\mathrm{f}}\right)\left(A / A_{\mathrm{f}}\right) e^{E / R^{\circ} T_{\mathrm{f}}-E / R^{\circ} T}$,

$\kappa_{2}=\left(\rho / \rho_{\mathrm{f}}\right)^{2}\left(\lambda / \lambda_{\mathrm{f}}\right)\left(k_{2} / k_{\mathrm{lf}}\right)$,

$\kappa_{3}=\left(\rho / \rho_{\mathrm{f}}\right)^{2}\left(\lambda / \lambda_{\mathrm{f}}\right)\left(6 p / R^{\circ} T\right)\left(k_{3} / k_{1 \mathrm{f}}\right)$,

and

$\kappa_{4}=\left(\rho / \rho_{\mathrm{f}}\right)^{2}\left(\lambda / \lambda_{\mathrm{f}}\right)\left(3 p / 2 R^{\circ} T\right)\left(k_{4} / k_{1 \mathrm{f}}\right)$,

where $p$ is pressure and $k_{i}$ denotes the conventional specific reaction-rate constant for step $i$. The ratios $\kappa_{i}$ are independent of $p$ for the two-body steps and proportional to $p$ for the three-body steps. In general the $\kappa_{i}$ vary with temperature as well as with composition (to the extent that the product $\lambda \rho^{2}$ varies with composition), but for $i=$ $(2,3,4)$ these variations are presumed to be weak enough for the $\kappa_{i}$ to be treated as constants in the reaction zone (in which region the subscript $f$ will be applied to $k$ 's but, for brevity, not to $\kappa$ 's). The Arrhenius temperature dependence is written explicitly in (12) to emphasize the fact that $\kappa_{1}$ varies strongly with $T$ and thereby is expected to cause a unique burning velocity to exist in an asymptotic sense.

Boundary conditions for (5), (6), and (7) are $\theta$ $=0, Y_{1}=0$, and $Y_{3}=Y_{3 \mathrm{u}}$ at $x=-\infty$ and $\theta=$
1, $Y_{1}=0$, and $Y_{3}=0$ at $x=+\infty$. The large expansion parameter of the problem is the relevant Zel'dovich number.

$\beta=E\left(T_{\mathrm{f}}-T_{\mathrm{u}}\right) /\left(R^{\circ} T_{\mathrm{f}}^{2}\right)$,

in terms of which the expansion of $\kappa_{1}$ is

$\kappa_{1}=\exp \left[\beta\left(\theta-\theta_{\mathrm{f}}\right) / \theta_{\mathrm{f}}+\cdots\right]$,

where $\theta_{\mathrm{f}}=3 W c_{\mathrm{p}}\left(T_{\mathrm{f}}-T_{\mathrm{u}}\right) /\left(Q Y_{3 \mathrm{u}}\right)$. The objective is to find asymptotic expansions for $\theta_{\mathrm{f}}, \Lambda, \theta(x)$, $Y_{1}(x)$, and $Y_{3}(x)$, with $Y_{3 u}, \mu, L_{1}, L_{3}, \kappa_{2}, \kappa_{3}$, and $\kappa_{4}$ treated as constants.

A linear combination of (5), (6), and (7), representing a conservation equation for total enthalpy, can be integrated once, giving

$$
\begin{aligned}
\theta+\frac{3 Y_{1}}{\mu Y_{3 \mathrm{u}}}+\frac{Y_{3}-Y_{3 \mathrm{u}}}{Y_{3 \mathrm{u}}}= & \frac{d \theta}{d x}+\frac{3}{L_{1} \mu Y_{3 \mathrm{u}}} \frac{d Y_{1}}{d x} \\
& +\frac{1}{L_{3} Y_{3 \mathrm{u}}} \frac{d Y_{3}}{d x},
\end{aligned}
$$

which may be used to replace either (5), (6), or (7), if convenient. For $L_{1}=L_{3}=1$ a further integration of (18) yields $\theta+Y+Z=1$, where

$Y \equiv Y_{3} / Y_{3 u}, \quad Z \equiv(3 / \mu)\left(Y_{1} / Y_{3 u}\right)$

These observations demonstrate that in general in the problem there are only two independent differential equations involving reaction rates.

\section{COMBUSTION REGIMES}

Because of the large number of parameters in the formulation there is a wide variety of classes of problems that can be considered. Not all possibilities will be addressed. In particular, attention will be restricted to problems with $L_{1}$ and $L_{3}$ of order unity (often they will be set equal to unity for simplicity) and to problems with $Y_{3 u}$ of order unity. The main influences to be investigated are those of the rate ratios: $\kappa_{2}, \kappa_{3}$, and $\kappa_{4}$. While $\mu$ will be treated as being of order unity, a few comments may first be made about potential simplifications that arise as $\mu \rightarrow 0$ or as $\mu \rightarrow 1$. 


\subsection{Influences of the Energetic Parameter}

For $\mu=1$ Eq. (10) shows that $D=0$, and therefore the intermediate interacts only kinetically, behaving energetically like the reactant; although two terms disappear from (5) in this limit, there is no real simplification in the structure of the problem or the regimes encountered. As $\mu$ $\rightarrow 0, D / Q \rightarrow \infty$ from (10), and energetics then restrict $Y_{1}$ to small values, suggesting that, in a first approximation, a steady-state approximation may apply for $O$, seen from (6) to be given by

$Y_{1}\left(\kappa_{2} Y_{3}+\kappa_{3} Y_{1}+\kappa_{4} Y_{2}\right)=\kappa_{1} Y_{3} Y_{4}$,

which simplifies to $Y_{1}=\kappa_{1} Y_{3}\left(1 / 2-Y_{3} / 6\right) /\left[\kappa_{4}\right.$ $+\left(\kappa_{2}-\kappa_{4}\right) Y_{3}$ ] unless $\kappa_{3}$ is unusually large. The problem then reduces to that of a one-reactant, one-step process, although the rate expression remains sufficiently complicated that a number of different regimes still occur, depending on relative magnitudes of $\kappa_{2}, \kappa_{3}$, and $\kappa_{4}$. These regimes are similar to some of those to be discussed for $\mu$ of order unity. Formal asymptotic developments for small values of $\mu$ could be interesting in revealing special attributes but are not pursued herein.

\subsection{The Steady State for $O$}

Always there will exist a reaction zone somewhere in which $\kappa_{1}$ is of order unity (i.e., $\theta-\theta_{\mathrm{f}}$ is of order $\left.\beta^{-1}\right)$, by definition. From (6) it follows that if $\kappa_{2}, \kappa_{3}$, or $\kappa_{4}$ is sufficiently large, then the steadystate approximation for $\mathrm{O}$ will be good in this zone, and with $\mu$ of order unity, $Z \ll Y$ there. In this zone, it is then found from (5) and (7) that a reactive-diffusive balance is attained, that $Y$ is of order $\beta^{-1}$, and that a convective-diffusive zone precedes this reaction zone. ${ }^{2}$ If the steady state is caused by a sufficiently large value of $\kappa_{3}$ alone, by

\footnotetext{
${ }^{2}$ This excludes a peculiar regime in which the source of the steady state is a large value of $\kappa_{4}$, with $\kappa_{2}$ and $\kappa_{3}$ negligible in the first reaction zone, so that reaction (1) attains partial equilibrium there. This zone is followed by increasing dissociation and eventually by heat release through (2) and/or (3). From the rate parameters for ozone [6] it is readily seen that this regime cannot occur (at least for $Y_{3 \mathrm{u}}>10^{-2}$, which in practice excludes it entirely), and it will not be considered further.
}

a sufficiently large value of $\kappa_{2}$ with either $\kappa_{3}$ or $\kappa_{4}$ also nonnegligible in this reaction zone, or by $\kappa_{2}, \kappa_{3}$, and $\kappa_{4}$ all being important in this reaction zone, then $\theta_{f}=1$, the reaction goes to completion in this zone, and the simple and well-known structure for a one-step reaction of large Zel'dovich number is recovered, although with a somewhat complicated reaction-rate expression. From rate parameters [6] it can be shown that this situation arises for the ozone decomposition flame only if $Y_{3 u}$ is unreasonably small or pressures are very high (typically $\geq 100 \mathrm{~atm}$ ).

Of special interest in ozone decomposition is the regime in which the steady state is associated with a sufficiently large value of $\kappa_{2}$, with $\kappa_{3}$ and $\kappa_{4}$ both negligible in this reaction zone. Then from (19), (8), and (9) it is seen that the steady state gives $Y_{1}$ $=\kappa_{1} / 2 \kappa_{2}$ in this reaction zone, which increases continually as $\theta$ increases. Since (17) shows that $\kappa_{1}$ approaches unity at the downstream end of this zone (by definition), imposition of zero gradients and of $\mathrm{R}$ depletion there gives, from (18), $\theta_{\mathrm{f}}=1$ $-3 /\left(2 \mu Y_{3 \mathrm{u}} \kappa_{2}\right)$ as the expression for determining $\theta_{f}$; in physical variables, the zero gradient alone gives

$T_{\mathrm{f}}=T_{\mathrm{u}}+\frac{Q Y_{3 \mathrm{u}}}{3 W c_{\mathrm{p}}}-\frac{Q+D}{W c_{\mathrm{p}}} Y_{1 \mathrm{f}}$,

while the full result just derived gives

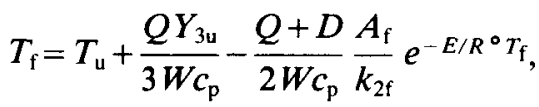

which always possesses a unique solution for $T_{\mathrm{f}}$. Downstream from this primary reaction zone is a broader recombination zone in which (3) and/or (4) occur, producing a further increase of temperature until $\theta=1$. It may be inferred from (19), for example, that the existence of this regime is somewhat unique to reactions with active intermediaries and would not arise if, for example, reaction (2) were replaced by a sequential mechanism that did not involve a collision with $R$. The regime involves an overshoot of the intermediate concentration to superequilibrium values and may be anticipated to be prevalent for flames involving chain reactions. The earliest clear identification of 
this regime for the ozone flame appears to have been made by von Kármán and Penner [10]. With improved kinetic data it is found to occur for ozone flames (at typical pressures and initial temperatures of interest) only if the initial ozone mole fraction is below about 0.4 [6].

\subsection{Merged Regimes}

It has been observed that with the steady-state approximation $Y$ is of order $\beta^{-1}$ in the reaction zone. This result may be used in (6) to define more precisely how large $\kappa_{2}, \kappa_{3}$, or $\kappa_{4}$ must be for the steady state to apply. For example, if large values of $\kappa_{2}$ are responsible for the steady state (with $\kappa_{3}$ and $\kappa_{4}$ negligible in this reaction zone), then $K \gg$ $\mathrm{I}$ is found to be the steady-state criterion, where

$K \equiv 2 \mu \kappa_{2} L_{1} Y_{3 \mathbf{u}} / 3 \beta$

which is influenced mainly by the ratio of the specific reaction-rate constants of steps (1) and (2) at temperature $T_{\mathrm{f}}$. Reduction of the important $\kappa_{\mathrm{i}}$ until the criterion for the steady state no longer is satisfied, e.g., until $K$ is of order unity, results in the necessity of considering two independent differential equations in the primary reaction zone. For ozone this has been termed the "merged regime," and analyses of it have been developed $[6,7]$. Further considerations of the merged regime for the ozone flame, including investigations of limiting behaviors for large and small values of $K$, are presented in Appendix A.

A straightforward situation (not very pertinent to ozone) in which two independent differential equations are needed to describe the structure of a thin reaction zone is that in which $\kappa_{2} / \beta, \kappa_{3} / \beta$, and $\kappa_{4}$ (as well as $L_{1}, L_{3}, Y_{3 u}$, and $\mu$ ) all are of order unity; in this regime $\theta_{\mathrm{f}}=1, Y_{3}$ and $Y_{1}$ both are of order $\beta^{-1}$ in the reaction zone (which maintains a reactive-diffusive balance in the first approximation), and all of the reaction-rate terms remain of the same order in (5), (6), and (7). It may in fact be seen in general that (with the other parameters of order unity) having $\kappa_{2} / \beta, \kappa_{3} / \beta$, or $\kappa_{4}$ of order unity or larger is sufficient to assure the occurrence of either a merged or a steady-state regime and to keep the maximum $\mathrm{O}$ mole fraction of order $\beta^{-1}$ or less. With $\kappa_{2} / \beta$ of order unity a merged regime with $\theta_{\mathrm{f}}=1$ and $Y_{1}$ of order $\beta^{-1}$ continues to prevail (but with simpler kinetics) if $\kappa_{3} / \beta$ or $\kappa_{4}$ is allowed to become arbitrarily small; if they both become small, then $\theta_{\mathrm{f}}<1$ and a larger recombination zone follows the primary reaction zone. With $\kappa_{3} / \beta$ of order unity, again a merged regime (with simpler kinetics) continues to prevail (with $\theta_{\mathrm{f}}=1$ and $Y_{1}$ of order $\beta^{-1}$ ) if $\kappa_{2} / \beta$ is decreased below unity. However, decreasing the order of magnitude of $\kappa_{2} / \beta$ below unity, with $\kappa_{3} / \beta$ and $\kappa_{4}$ restricted to be of the same order of magnitude as $\kappa_{2} / \beta$ or less, leads to a new regime that at least a priori could possess relevance to the ozone decomposition flame. This regime arises when $K \ll 1$, for the $K$ defined in (22).

\subsection{Two-Zone Regimes}

Decreasing $\kappa_{2} / \beta$ below unity, with $\kappa_{3} / \beta$ and $\kappa_{4}$ sufficiently small, is seen from (5) to tend to increase $Y_{1}$ in the reaction zone, since the requirement of nonnegative net heat-release rates there imposes the restriction $(1+\mu) \kappa_{2} Y_{1} \geq(1-$ н) $\kappa_{1} Y_{4}$. The next (and last) possible distinguished ordering for $Y_{1}$ in the reaction zone is $Y_{1}$ of order unity, corresponding to $\kappa_{2}$ of order unity. ${ }^{3}$ Since the energetics fundamentally are imposing these orderings, it is necessary that in this reaction zone the equality

$$
\begin{aligned}
(1-\mu) \kappa_{1} Y_{3} Y_{4}= & (1+\mu) \kappa_{2} Y_{1} Y_{3}+\kappa_{3} Y_{1}^{2} \\
& +(1-\mu) \kappa_{4} Y_{1} Y_{2}
\end{aligned}
$$

will apply in the first approximation [i.e., $(1+$ н) $\kappa_{2} Y_{1}=(1-\mu) \kappa_{1} Y_{4}$ when $\kappa_{3}$ and $\kappa_{4}$ are negligible]. From (5) it is evident that (23) corresponds to a steady-state approximation for the temperature in this reaction zone. A way to look at this result when $\kappa_{3}$ and $\kappa_{4}$ are negligible is

\footnotetext{
${ }^{3}$ If $\kappa_{2}$ were any smaller (with $\kappa_{3}$ and $\kappa_{4}$ negligible), then this reaction zone would be endothermic and could not control the burning velocity. In fact, step (1) would proceed already at the initial temperature (i.e., $T_{\mathrm{f}} \rightarrow T_{\mathrm{u}}$ ), and a quasi-steady burning velocity would not exist for the adopted mechanism because steps (2), (3), and (4) have been assigned zero activation energies.
} 
to observe from (5) and (6) that the ratio $\kappa_{2} Y_{1} / \kappa_{1} Y_{4}$ is restricted to a somewhat narrow range of values of order unity, viz., $(1-\mu) /(1+\mu) \leq \kappa_{2} Y_{1} /$ $\kappa_{1} Y_{4} \leq 1$, the upper limit being established by the requirement that the net rate of production of $\mathrm{O}$ be nonnegative and the lower by the requirement of a nonnegative net rate of heat release; equality at the upper limit defines the steady-state approximation for $\mathrm{O}$ and equality at the lower limit the steadystate approximation for $\theta$. As $\mu$ approaches zero (23) approaches (19), and the two steady states become identical; $Y_{1}$ cannot increase to values of order unity if $\mu$ is too small.

Whenever neither $O$ nor $R$ is in a steady state and $\kappa_{3}$ is negligible in the reaction zone, it is found by comparing (6) and (7) that the reaction-rate terms are of the same order of magnitude in each and that therefore the changes in $Y_{1}$ and $Y_{3}$ must be of the same order of magnitude (in merged regimes this is achieved by having $Y_{1}$ and $Y_{3}$ both of order $\beta^{-1}$ ). It follows that when $Y_{1}$ is of order unity in the reaction zone, $Y_{3}$ also must be of order unity there. To accommodate these higher reactant concentrations the reaction zone must become comparatively broader, so that all three effects in (6) and (7)-convection, diffusion and reactionare important there; attempts to impose narrower reaction zones result in concentration gradients at the boundaries that are too steep for matching. With $Y_{1}$ of order unity in the reaction zone, upstream diffusion of $O$ into the preheat zone no longer can be neglected, and since $\kappa_{2}$ is constant, the $O$ that diffuses into the preheat zone will react with $R$ there by (2), thereby causing the preheat zone also to develop a convective-reactive-diffusive balance. Therefore this type of regime may be called a "two-zone regime," in which some of the $O$ produced in the downstream reaction zone by (1) diffuses forward to release heat by (2) in an upstream reaction zone that formerly was merely the convective-diffusive preheat zone. The possibilities for the existence flame structures in which essential roles are played by active species that are produced in hot downstream regions and that diffuse upstream to react exothermically in cooler regions have been well recognized and discussed in the early literature [10]. Compatibility of this concept with activation-energy asymptotics appears to need a structure like that of the two-zone regime identified here.

\subsection{Thicknesses of Zones}

Figures 1 and 2 are qualitative illustrations of flame structures in the merged and two-zone regimes, respectively. They both correspond to conditions under which $\kappa_{3}$ and $\kappa_{4}$ are both sufficiently small for the recombination zone to exist $\left(\theta_{\mathrm{f}}<1\right)$. In Fig. 2 the convective-reactivediffusive zone that maintains a steady state for temperature is identified as the generation zone since this is the only region in which the intermediate $\mathrm{O}$ is generated. Because of the exothermicity of (2) in the preheat zone, this zone has been relabled the exothermic zone. Heat release can extend all the way to the cold boundary in Fig. 2 without

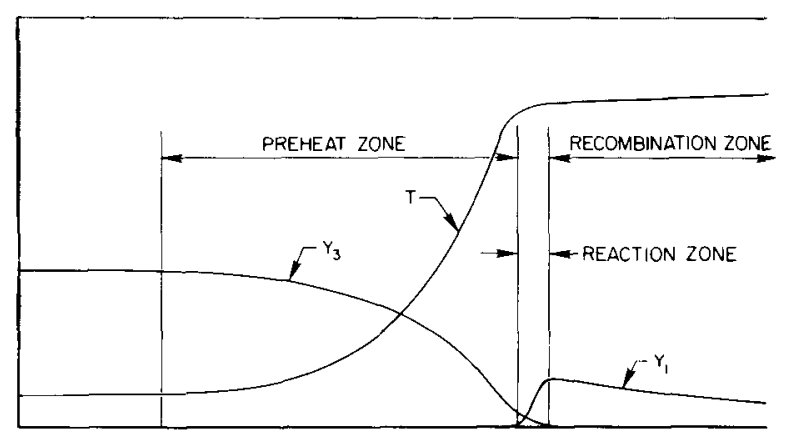

Fig. 1. Illustration of the structure of the merged regime. 


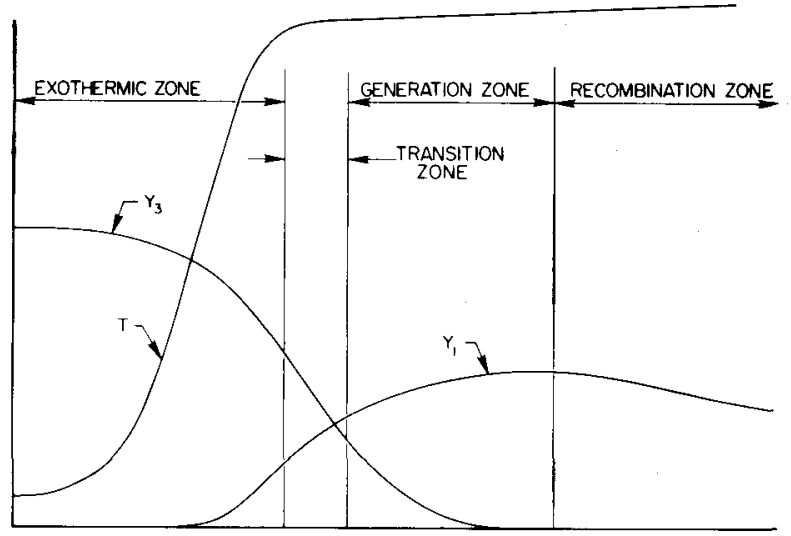

Fig. 2. Illustration of the structure of the two-zone regime.

violating activation-energy asymptotics or interfering with the existence of a burning velocity because the release occurs in a step with zero activation energy, involving as an essential reactant a species (O) produced elsewhere (downstream) in a step with a large Zel'dovich number.

In the nondimensional $x$ coordinate the thickness of the preheat zone in Fig. 1 is of order unity, the thickness of the reaction zone is of order $\beta^{-1}$, and the thickness of the recombination zone is of order $\beta^{-2}\left(\kappa_{3} / \kappa_{2}+\kappa_{4}\right)^{-1}$, presumed to be greater than unity. In Fig. 2 the thickness of the recombination zone is of order $\left(\kappa_{3}+\kappa_{4}\right)^{-1}$, the thicknesses of the generation and exothermic zones are each of order unity, and there is a transition zone having a thickness of order $\beta^{-1 / 2}$ between these two. The burning-rate eigenvalue $\Lambda$ in (5), (6), and (7) is of order $\beta^{2}$ in steady-state and merged regimes but of order unity in two-zone regimes. Since it is seen from (11) that the burning velocity varies inversely as the square root of $\Lambda$, the burning velocity may be expected to be appreciably higher in the two-zone regime. Therefore the physical distances are proportionally smaller in the twozone regime; Figs. 1 and 2 illustrate nondimensional distances, not physical distances. Experimentally it would be found that for Fig. 1 the flame is thicker but the chemistry begins only after most of the temperature increase has occurred, while for Fig. 2 the flame is thinner but the chemistry extends throughout the entire flame.

\subsection{Regimes for the Recombination Zone}

While (3) and (4) both may be important in the recombination zone, equality of the recombination rates by each at the flame temperature $T_{\mathrm{f}}$ defines a rough dividing line between dominance of one or the other of these steps in recombination. Thus, with

$J \equiv \kappa_{3} Y_{\mathrm{If}} / \kappa_{4} Y_{2 \mathrm{f}}$

step (3) may be expected to be dominant for $J \gg 1$ and step (4) for $J \ll 1$. With this definition it is found that, approximately, for steady-state or merged regimes $J=\kappa_{3} /\left(2 \kappa_{2} \kappa_{4}\right)$, for merged regimes alternatively $J \approx \kappa_{3} / \beta_{4}$, and for twozone regimes $J \approx \kappa_{3} / \kappa_{4}$. In the recombination zone it is typical that $Y_{3} \ll Y_{1}$ so that, by comparing (6) and (7), it is found that a steady-state approximation for R can be applied. From (7) it is then found that in the recombination zone

$Y_{3}=\kappa_{4} Y_{1} Y_{2} /\left(\kappa_{2} Y_{1}+\kappa_{1} Y_{4}\right)$

in a first approximation. This result is not very important for $J \gg 1$ because then $\mathrm{R}$ is kinetically negligible and may be taken to be essentially zero in the recombination zone, but for $J \ll 1$ it provides the essential path to the formation of $\mathrm{P}$ in the recombination. Essentially, step (4) is followed by step (2) in the steady state for R, so that the effective nondimensional rate of recombination 
of $\mathrm{O}$ to form $\mathrm{P}$ is

$\kappa_{3} Y_{1}^{2}+2 \kappa_{4} Y_{1} Y_{2}\left[\kappa_{2} Y_{1} /\left(\kappa_{2} Y_{1}+\kappa_{1} Y_{4}\right)\right]$

in which the last factor accounts for a reduction in the rate with increasing temperature and decreasing $Y_{1}$ through onset of step (1) as a competitor to (2). The importance of these reactions involving $R$ $\left(\mathrm{O}_{3}\right)$ near the hot boundary for the ozone flame was recognized by von Kármán and Penner [10].

\subsection{Summary of Regimes}

Table 1 summarizes rough orders of magnitudes of rate parameters for various regimes when step (2) is important in the primary reaction zone.

\section{ANALYSIS OF THE TWO-ZONE STRUCTURE}

The manner in which the two-zone structure develops as $k_{1 \mathrm{f}}$ increases may be seen from the analysis of the merged regime for the limit $K \rightarrow 0$, as given in Appendix $\mathrm{A}$. When $K$ becomes sufficiently small the reaction zone no longer is thin, and it is convenient to work with the variables $Y$ and $Z$ defined after (18). For simplicity of presentation, $\kappa_{3}$ and $\kappa_{4}$ will be assumed to be small enough to be negligible except in the recombination zone, which will not be analyzed here. In the two-zone regime there are two distinguished locations within the flame, viz., the downstream and upstream ends of the generation zone, the last of which also may be identified as the downsteam end of the exothermic zone, or simply as the location of the transition zone. It aids in clarification to have two different subscripts to

\section{TABLE 1}

Orderings for Regimes

\begin{tabular}{ccc}
\hline$\kappa_{2}$ & Regime & $\begin{array}{c}\text { Condition for Distinct } \\
\text { Recombination Zone }\end{array}$ \\
\hline$>\beta$ & Steady-state & $\kappa_{3}<\kappa_{2}^{2} / \beta, \kappa_{4}<\kappa_{2} / \beta$ \\
$\beta$ & Merged & $\kappa_{3}<\beta, \kappa_{4}<1$ \\
1 & Two-zone & $\kappa_{3}<1, \kappa_{4}<1$ \\
\hline
\end{tabular}

distinguish these two locations, and since the subscript $f$ has been used in (20) to identify conditions that are the same as those applied at the downstream end of the generation zone, it seems convenient to retain $f$ for that purpose and to introduce the subscript $t$ to identify conditions at the transition layer. Analysis of the two-zone structure is facilitated by employing transitionlayer conditions as reference states for measurement of rates. Therefore in (11)-(16) the subscript $f$ may simply be replaced by $t$ for the purposes of the present analysis. Since $\theta_{\mathrm{f}}-\theta_{\mathrm{t}}$ will be of order $\beta^{-1}$, the difference is asymptotically small in formulas like (16), but it is important in Arrhenius factors of rates.

The function

$F \equiv 3 Y_{4} \kappa_{1} /\left(\mu Y_{3 u} \kappa_{2}\right)$

is now introduced, and from (12) and (13) it may then be shown that

$F=B e^{-\beta\left(1-\theta / \theta_{\mathrm{t}}\right) /\left[1-\alpha\left(1-\theta / \theta_{\mathrm{t}}\right)\right]}$,

where

$$
\begin{gathered}
B \equiv\left[3 Y_{4} A /\left(\mu Y_{3 \mathrm{u}} k_{2}\right)\right] e^{-E / R^{\circ} T_{t},} \\
\alpha \equiv\left(T_{t}-T_{\mathrm{u}}\right) / T_{t},
\end{gathered}
$$

and where use has been made of (16) and of the definition of $\theta$. Here $B$ is assumed to vary at most slowly with $\theta$, so that the major dependence of $F$ upon $\theta$ is exhibited explicitly in (26). For simplicity the further approximations $L_{1}=L_{3}=1$ and $\rho^{2} \lambda k_{2}=$ constant are adopted, whence (6) and (7) become

$$
d Y / d x=d^{2} Y / d x^{2}-\mu \Lambda_{0} Y(F+Z)
$$

and

$$
d Z / d x=d^{2} Z / d x^{2}+\Lambda_{0} Y(F-Z),
$$

where the modified constant eigenvalue of order unity is

$$
\Lambda_{0} \equiv Y_{3 \mathrm{u}} \kappa_{2} \Lambda / 3=\frac{Y_{3 \mathrm{u}}\left(\rho^{2} \lambda k_{2}\right)_{1}}{3 W c_{\mathrm{p}} \rho_{\mathrm{t}}^{2} v_{\mathrm{t}}^{2}},
$$

in the last equality of which (11) and (13) have 
been employed. The relationship

$\theta=1-(Y+Z)$,

derived after (18), may be used for $\theta$ in (26) to obtain a problem posed in terms of $Y$ and $Z$ alone. The boundary conditions for (27) and (28) are $Y(-\infty)=1, Z(-\infty)=0, Y(\infty)=0$, and $d Z /$ $d x \rightarrow 0$, i.e., $Z \rightarrow Z_{\mathrm{f}}=$ constant, as $x \rightarrow \infty$.

It is convenient to place the transition layer at $x$ $=0$. Then in the exothermic zone $(x<0) F$ is seen from (26) to be exponentially small and therefore negligible in (27) and (28). The differential equation for $Y-\mu Z$ therefore lacks a chemical source term; it is linear and can readily be integrated twice, yielding

$Z=\left[C e^{x}-(1-Y)\right] / \mu$,

where the boundary conditions at $x=-\infty$ have been applied, and the integration constant $C$ is to be found by matching at the transition layer. Use of (31) in (27) gives

$d Y / d x=d^{2} Y / d x^{2}-\Lambda_{0} Y\left[C e^{x}-(1-Y)\right]$

as the remaining equation to be integrated for $x<$ 0 , subject to $Y(-\infty)=1, Y(0)=Y_{\mathrm{t}}$, and $(d Y /$ $d x)=Y_{\mathrm{t}}^{\prime}$ at $x=0$ with $Y_{\mathrm{t}}$ and $Y_{\mathrm{t}}^{\prime}$ constants as yet unknown.

In the generation zone $(x>0) F$ would be exponentially large if the order of $\left(\theta-\theta_{t}\right) / \theta_{1}$ were larger than $\beta^{-1}$. Since this would be unacceptable (and could occur only farther downstream, in the recombination zone), $\beta\left(\theta-\theta_{\mathrm{t}}\right) / \theta_{\mathrm{t}}$ must be of order unity here, and (30) becomes, in the first approximation,

$Z=\left(1-\theta_{t}\right)-Y$.

Since changes in $\theta$ now are of order $\beta^{-1}$ here, it follows from (30) that the derivatives that appear when (27) and (28) are added are of order $\beta^{-1}$, so that, in the first approximation,

$F=Z(1+\mu) /(1-\mu)$,

which defines the steady state for temperature in the present variables. Use of (33) and (34) in (27) gives the differential equation

$d Y / d x=d^{2} Y / d x^{2}-\Lambda_{0} Y\left(1-\theta_{\mathrm{t}}-Y\right)[2 \mu /(1-\mu)]$,

to be integrated for $x>0$ subject to $Y(\infty)=0$, $Y(0)=Y_{\mathrm{t}}$, and $(d Y / d x)=Y_{\mathrm{i}}^{\prime}$ at $x=0$. That these latter conditions are the same as those following (32) is an immediate consequence of the transition-zone equation for $Y$, found by stretching $x$ in (27) to be simply $d^{2} Y / d x^{2}=0$ in the first approximation.

From (32) and (35) it is seen that a continuous function $Y$ with a continuous first derivative is to be sought, obeying

$$
\begin{aligned}
\frac{d^{2} Y}{d x^{2}}= & \frac{d Y}{d x}+\Lambda_{0} Y \\
& \cdot\left\{\begin{array}{ll}
\left(\frac{2 \mu}{1-\mu}\right)\left(1-\theta_{\mathrm{t}}-Y\right) & \text { for } x>0 \\
C e^{x}-(1-Y) & \text { for } x<0
\end{array}\right\}
\end{aligned}
$$

with $Y(-\infty)=1$ and $Y(\infty)=0$. The transitionlayer equation for $Z$, obtained from (28), similarly shows that $Z$ must be continuous with a continuous first derivative at $x=0$. From (31) and (33) these requirements are seen to give

$C=(1+\mu)\left(1-Y_{\mathrm{t}}\right)-\mu \theta_{\mathrm{t}}=-Y_{\mathrm{t}}{ }^{\prime}(1+\mu)$.

These two additional equations serve to determine the parameters $C$ and $\Lambda_{0}$ from the solution to (36) for given values of $\mu$ and $\theta_{\mathrm{t}}$.

The solution to this quadratically nonlinear problem was obtained numerically by integration employing a phase plane. For $x>0$ the variables $X=[2 \mu /(1-\mu)] \Lambda_{0} Y$ and $X^{\prime}=d X / d x$ are helpful, so that with $a \equiv[2 \mu /(1-\mu)] \Lambda_{0}\left(1-\theta_{t}\right)$, the equation $d X^{\prime} / d X=1+X(a-X) / X^{\prime}$ is derived, which is integrated numerically, for selected values of $a$, subject to $X^{\prime}=0$ at $X=0$, starting with the expansion $X^{\prime}=-(\sqrt{1+4 a}-$ 1) $X / 2+\cdots$, to obtain permissible combinations of $X_{\mathrm{t}}^{\prime}$ and $X_{\mathrm{t}}$ for use at $x=0$. For $x<0$, the variables $\bar{X}=\Lambda_{0} Y, \bar{X}^{\prime}=d \bar{X} / d x$, and $U=$ $\Lambda_{0}(Y-\mu Z)$ are helpful, so that $d \bar{X}^{\prime} / d \bar{X}=1+$ $\bar{X}(\bar{X}-U) / \bar{X}^{\prime}$ and $d U / d \bar{X}=\left(U-\Lambda_{0}\right) / \bar{X}^{\prime}$ 
could have been integrated numerically from $\bar{X}=$ $X_{\mathrm{t}}(1-\mu) / 2 \mu$, starting with $\bar{X}^{\prime}=X_{\mathrm{t}}^{\prime}(1-\mu) / 2 \mu$ and $U=U_{\mathrm{t}} \equiv\left[X_{\mathrm{t}}(1+\mu) / \mu-a\right](1-\mu) / 2$, adjusting the selections of $X_{1}$ and $\Lambda_{0}$ to obtain $\bar{X}^{\prime}$ $\rightarrow 0$ appropriately and $U=\Lambda_{0}$ at $\bar{X}=\Lambda_{0}$, the cold boundary. In fact, because of the singular character of the solution at the cold boundary in the phase plane, it proved to be simpler to integrate $d^{2} \bar{X} / d x^{2}=d \bar{X} / d x+\bar{X}(\bar{X}-U)$, with $U=U_{\mathrm{t}}-U_{\mathrm{t}}^{\prime}\left(1-e^{x}\right)$ and $U_{\mathrm{t}}^{\prime}=X_{\mathrm{t}}^{\prime}\left(1-\mu^{2}\right) / 2 \mu$, starting from $x=0$ to obtain $\bar{X}=U=\Lambda_{0}$ at $x=$ $-\infty$ by adjusting $X_{0}$ and $\Lambda_{0}$. The adjustments were performed by hand to obtain the representative results shown in Fig. 3. Corresponding curves (not shown) have also been calculated for $\mu=$ 0.414 and for $\mu=0.100$.

Also shown in Fig. 3 are dashed curves representing results of a two-term expansion for small values of $1-\theta_{\mathrm{t}} \equiv \epsilon$. In this limit, the reaction zones become thinner, and $\xi \equiv x / \epsilon$ becomes an appropriate independent variable, with $u \equiv Y / \epsilon$ the corresponding dependent variable of order unity. The constants $c \equiv(1-C) / \epsilon$ and $l \equiv \epsilon^{3} \Lambda_{0}$ become of order unity, and in these new variables (36) and (37) are

$$
\begin{aligned}
\frac{d^{2} u}{d \xi^{2}}=\epsilon & \frac{d u}{d \xi}+l u \\
& \cdot \begin{cases}(1-u) 2 \mu /(1-\mu) & \text { for } \xi>0 \\
u-c e^{\epsilon \xi}-\left(1-e^{\epsilon \xi}\right) / \epsilon & \text { for } \xi<0\end{cases}
\end{aligned}
$$

and

$c=(1+\mu) u_{\mathrm{i}}-\mu=\left[1+(1+\mu)(d u / d \xi)_{\mathrm{t}}\right] / \epsilon$.

The expansions $u=u_{1}+\epsilon u_{2}+\cdots, l=l_{1}+$ $\epsilon l_{2}+\cdots$, and $c=c_{1}+\epsilon c_{2}+\cdots$ may be introduced, and for $\xi>0$ integrations of equations obtained by collecting coefficients of successive powers of $\epsilon$ may be performed analytically to give, for example,

$l_{1}=(1-\mu) /\left[2 \mu(1+\mu)^{2} u_{1 \mathrm{t}}{ }^{2}\left(1-2 u_{1 \mathrm{t}} / 3\right)\right]$.

For $\xi<0$, the corresponding differential equations, e.g.,

$d^{2} u_{1} / d \xi^{2}=l_{1} u_{1}\left[u_{1}+\xi+\mu-(1+\mu) u_{1}\right]$

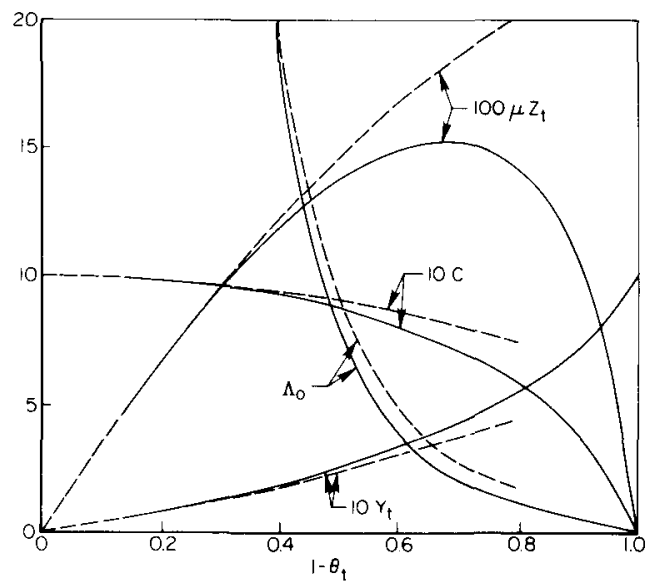

Fig. 3. Solutions for the two-zone structure, obtained from (36) and (37) (solid lines) and from the two-term expansion for $\theta_{1}$ near unity (dashed lines), for $\mu=0.573$.

subject to $\left(d u_{1} / d \xi\right)=-1 /(1+\mu)$ at $\xi=0$ (where $u_{1}=u_{1 \mathrm{t}}$ ) and to $\left(d u_{1} / d \xi\right)=-1$ at $\xi=$ $-\infty$, are of the same character as those encountered and discussed at the end of Appendix A, and therefore they must be integrated numerically, a task that was achieved by a shooting technique for various values of $\mu$ to obtain $l_{1}, l_{2}, c_{1}, c_{2}$ and $u_{1 \mathrm{t}}$, $u_{2 t}$. The results of the numerical integrations are displayed graphically in Fig. 4; for a few selected values of $\mu$ they are given in Table 2 . The etnry for $\mu=0.573$ enabled the dashed curves in Fig. 3 to be plotted. These curves are seen to agree reasonably well with the solid lines up to fairly large values of $\left(1-\theta_{t}\right)$ (e.g., to about $1-\theta_{t}=0.8$ for the $\Lambda_{0}$ curve). From (29) it therefore follows that the result

$v_{\mathrm{t}}^{2}=\frac{Y_{3 \mathrm{u}} \lambda_{\mathrm{t}} k_{2 \mathrm{t}}\left(1-\theta_{\mathrm{t}}\right)^{3}}{3 W c_{\mathrm{pt}}\left[l_{1}+l_{2}\left(1-\theta_{\mathrm{t}}\right)\right]}$,

with $l_{1}$ and $l_{2}$ obtained from Fig. 4 or Table 2, may be used with reasonable accuracy throughout most of the two-zone regime. The leading-order results for $\epsilon \rightarrow 0$ merge reasonably smoothly with those derived in Appendix A for $K \rightarrow 0$, as they should.

In the formulation that has been adopted here it was unnecessary to address the functional form of the solution for $\theta$ in the transition layer. If that is done through expansions of $Y$ and $Z$ in powers of $\beta^{-1 / 2}$ with $\psi=\beta\left(\theta-\theta_{\mathrm{t}}\right) / \theta_{\mathrm{t}}$ taken as a temperature 


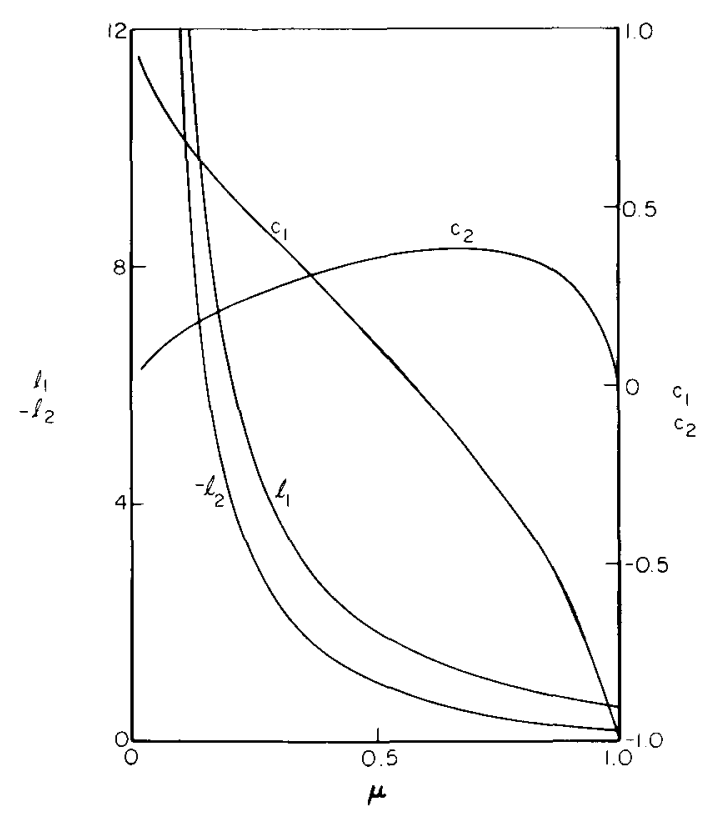

Fig. 4. Expansion coefficients in the two-zone structure for $\theta_{1}$ near unity.

variable or order unity, then a transition-layer problem for $\psi$ may be derived closely resembling that for $\phi$ derived in Appendix $A$ and solved following (A28). As $\epsilon \rightarrow 0$ the resulting transitionlayer solution for $\psi$ merges smoothly with that derived in Appendix A for $K \rightarrow 0$.

\section{AN ILLUSTRATIVE EXAMPLE}

To illustrate relationships among burning velocities calculated for various regimes Fig. 5 has been

\section{TABLE 2}

Coefficients for Expansion of the Two-Zone Structure as $\theta_{t}$ Approaches Unity

\begin{tabular}{crrrr}
\hline$\mu$ & \multicolumn{1}{c}{$l_{1}$} & \multicolumn{1}{c}{$l_{2}$} & \multicolumn{1}{c}{$c_{1}$} & $c_{2}$ \\
\hline 0.100 & 13.522 & -9.454 & 0.707 & 0.162 \\
0.300 & 3.687 & -2.302 & 0.404 & 0.286 \\
0.414 & 2.396 & -1.378 & 0.239 & 0.334 \\
0.500 & 1.831 & -0.983 & 0.113 & 0.362 \\
0.573 & 1.494 & -0.753 & 0.001 & 0.378 \\
0.700 & 1.091 & -0.488 & -0.207 & 0.385 \\
0.900 & 0.709 & -0.257 & -0.604 & 0.294 \\
\hline & & & & \\
\hline
\end{tabular}

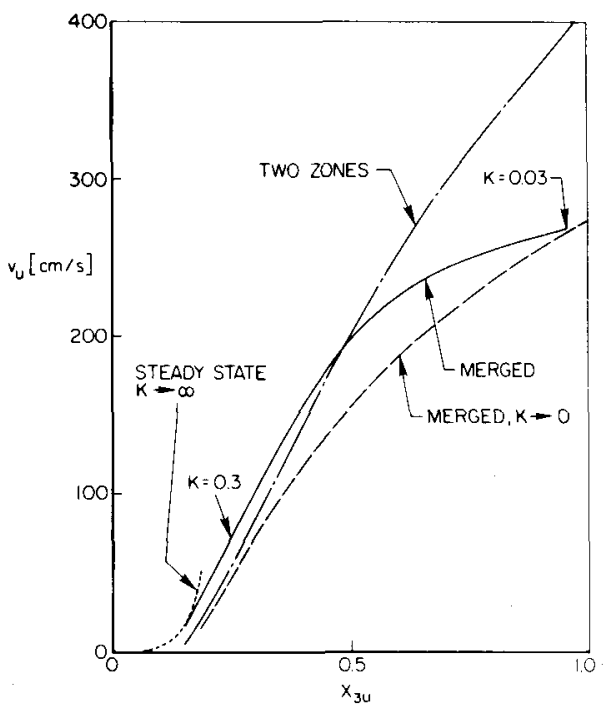

Fig. 5. Burning velocities in various regimes for an illustrative example.

prepared as a hypothetical example. This figure shows the dependence of the burning velocity on the initial reactant mole fraction $X_{3 \mathrm{u}}$, in terms of which $Y_{3 u}=(3 / 2) X_{3 u} /\left(1+X_{3 u} / 2\right)$. The parameters selected for this calculation were $p=1 \mathrm{~atm}$, $T_{\mathrm{u}}=300 \mathrm{~K}, Q=31.8 \mathrm{kcal} / \mathrm{mol}, \mu=0.1, W=$ $16 \mathrm{~g} / \mathrm{mol}, \lambda=4 \times 10^{-4} \mathrm{cal} / \mathrm{cm} \mathrm{s} \mathrm{K}, c_{\mathrm{p}}=0.25$ $\mathrm{cal} / \mathrm{g} \mathrm{K}, E=30 \mathrm{kcal} / \mathrm{mol}, A=10^{17} \mathrm{~cm}^{3} / \mathrm{mol} \mathrm{s}$, $k_{2}=10^{12} \mathrm{~cm}^{3} / \mathrm{mol} \mathrm{s}, k_{3}=k_{4}=0, L_{1}=L_{3}=1$, and $Y_{4}=1 / 2$.

The solid line is the result for the merged regime, obtained from (A4) and the analysis in Appendix A. Values of the parameter $K$ of (22) are shown at two points on this curve. Here $K$ becomes large only when $X_{3 u}$ is quite small, and the steady-state solution, obtained from (A4) with $V$ as found from (A5) and with $k_{1 \mathrm{f}}=A_{\mathrm{f}} e^{-E / R^{\circ} T_{\mathrm{f}}}$ calculated from (20) and (A6), is approached only in a narrow range at the left. The steady state is shown in Fig. 5 as a dotted line. The limiting formula for $K \rightarrow \infty$ is seen to apply fairly well even for $K \approx 1$ in this example. If the steady state is applied beyond its range of validity, then it predicts too large a value for the burning velocity.

As $K$ becomes sufficiently small the solid line approaches the dashed line labeled $K \rightarrow 0$, which was obtained from the expansion for small $K$ developed at the end of Appendix A. In this 
expansion $V$ varies inversely with $K$, the evaluation of which from (22) entails finding $T_{\mathrm{f}}$, which appears in $\beta$ of (16) and in $\kappa_{2}$ of (13) through $k_{1 \mathrm{ff}}$. Although (21) cannot be used for this purpose (since it assumes a steady state), (20) is more general and can be employed. This necessitates finding $Y_{1 \mathrm{f}}$, which through the definitions of $Z$ and $z$ with use of (A13) is found for $K \rightarrow 0$ to be given by $Y_{1 f}=[(1-\mu) /(1+\mu)] /\left(2 k_{2 f}\right)$. In view of (13), the result in (20) for $K \rightarrow 0$ therefore becomes

$$
\begin{aligned}
T_{\mathrm{f}}= & T_{\mathrm{u}}+\frac{Q Y_{3 \mathrm{u}}}{3 W c_{\mathrm{p}}}-\frac{Q+D}{2 W c_{\mathrm{p}}} \\
& \cdot\left(\frac{1-\mu}{1+\mu}\right) \frac{A_{\mathrm{f}}}{k_{2 \mathrm{f}}} e^{-E / R^{\circ} T_{\mathrm{f}}}
\end{aligned}
$$

which differs from (21) only in the factor $(1-\mu) /$ $(1+\mu)=D /(2 Q+D)$, which (in contrast to $K$ $\rightarrow \infty$ ) no longer is unity when $K \rightarrow 0 .{ }^{4}$ The dashed line giving the results for $K \rightarrow 0$ in Fig. 5 is seen to be approached by the solid line only for quite small values of $K$. Their difference is appreciable throughout most of the figure, the burning velocity for the $K \rightarrow 0$ limit always being less than that of the full merged-regime solution.

The broken line in Fig. 5 is the solution obtained using the expressions for the two-zone regime. In this regime the burning velocity $v_{u}=$ $\left(T_{\mathrm{v}} / T_{t}\right) v_{t}$ is found from $\Lambda_{0}$ of (29) through the analysis of the preceding section that gives $\Lambda_{0}(\mu$, $\theta_{1}$ ). A reasonable approximation to $v_{t}$ for much of this regime is given by (40). The value of $\theta_{1}$ is obtained from (26) and (34) evaluated at $x=0$, which give $B_{\mathrm{t}}=Z_{\mathrm{t}}(1+\mu) /(1-\mu)$, or in view of the definition of $B$,

$A_{\mathrm{t}} \mathrm{e}^{-E / R^{\circ} T_{\mathrm{t}}}=\frac{Y_{3 \mathrm{u}} k_{2 \mathrm{t}}}{3 Y_{4 \mathrm{t}}}\left(\frac{1+\mu}{1-\mu}\right) \mu Z_{\mathrm{t}}$,

where $T_{\mathrm{t}}=T_{\mathrm{u}}+\theta_{\mathrm{t}} Q Y_{3 \mathrm{u}} /\left(3 W c_{\mathrm{p}}\right)$. The solution to (36) relates $\mu Z_{1}$ in (42) to $\theta_{t}$ through (33) (see Fig.

\footnotetext{
${ }^{4}$ At intermediate values of $K$, to obtain results on the solid line in Fig. 5, the factor $(1-\mu) /(1+\mu)$ in (4I) effectively is replaced by a function $f(\mu, K)$, which varies monotonically with $K$, from $(1-\mu) /(1+\mu)$ at $K=0$ to unity at $K=\infty$ and which is obtained by calculating $Y_{\text {If }}$ from the results [6] of the analysis of the merged regime.
}

3 , for example), thereby enabling $\theta_{t}$ to be recovered from (42). A correspondence between (41) and (42) can be developed by evaluating (34) at $x$ $=\infty$ to obtain $F_{\mathrm{f}}=Z_{\mathrm{f}}(1+\mu) /(1-\mu)$, which in view of (26) is

$$
A_{\mathrm{f}} e^{-E / R^{\circ} T_{\mathrm{f}}}=\left(\mu Y_{3 \mathrm{u}} k_{2 \mathrm{f}} / 3 Y_{4 \mathrm{f}}\right)\left(\frac{1+\mu}{1-\mu}\right)\left(1-\theta_{\mathrm{f}}\right) \text {, }
$$

where (33) evaluated at $x=\infty$ has been employed to write $Z_{\mathrm{f}}=1-\theta_{\mathrm{t}} \approx 1-\theta_{\mathrm{f}}$ on the right-hand side. Substituting the definition of $\theta$ into (43) and then putting $Y_{4 \mathrm{f}}=1 / 2$ (the value approached as $Y_{1 \mathrm{f}}$ or $1-\theta_{\mathrm{f}}$ approaches zero) gives (41).

The broken curve in Fig. 5 is seen to cross the solid curve and to merge with the dashed curve at relatively small $X_{3 \mathrm{u}}$. This latter agreement is to be anticipated from the correspondence of the limit $K$ $\rightarrow 0$ of the merged regime with the limit $\epsilon \rightarrow 0$ of the two-zone regime. The burning velocity for the two-zone solution always exceeds that obtained from the limit $K \rightarrow 0$ of the merged regime. In this example burning velocities for the two-zone regime do not nicely approach those for the full solution of the merged regime. If $\beta$ were large enough, then a continuous evolution would be observed, from the steady state, to the fully merged regime, to the merged regime for $K \rightarrow 0$, to the two-zone regime. Here instead the two-zone solution intersects the full-merged solution somewhat sharply. Since the two-zone solution is the more accurate one when it predicts a higher burning velocity than the merged solution, a procedure for computation of $v_{u}$ is to calculate solutions for both the fully merged regime and the two-zone regime, then select the larger of the two burning velocities. From (A4) and (40), this criterion can be expressed approximately by saying that the two-zone solution is to be employed if

$k_{2 \mathrm{f}}>k_{\mathrm{if}} \frac{3\left[l_{1}+l_{2}\left(1-\theta_{\mathrm{f}}\right)\right]}{2 \beta^{2} Y_{3 \mathrm{u}}\left(1-\theta_{\mathrm{f}}\right)^{3}} V^{2}$.

A more compact form of this criterion is obtained by eliminating $k_{1 \mathrm{f}}$ and $k_{2 \mathrm{f}}$ through (43), viz.,

$$
\mu(1+\mu)\left[l_{1}+l_{2}\left(1-\theta_{\mathrm{f}}\right)\right] V^{2}<(1-\mu) \beta^{2}\left(1-\theta_{\mathrm{f}}\right)^{2} .
$$


Use of (44) involves finding $\theta_{\mathrm{f}}$ from the two-zone solution as outlined above and $V$ from the mergedregime solution of Appendix A.

It seems reasonable that the regime selection criterion should be that the regime having the higher burning velocity prevails. Penetration of the exothermicity into the preheat zone should occur only if it increases the burning velocity above the value for no such penetration. Corresponding reasoning does not apply to the steadystate approximation for $\mathrm{O}$, which, in a sense, is merely a special case of the merged regime. When the steady state fails the true $O$ concentration is less than that calculated for the steady state, and therefore the steady state gives values too high for overall reaction rates and burning velocities.

\section{COMPARISONS FOR THE OZONE FLAME}

For the ozone decomposition flame the energies $Q$ and $D$, approximate values for which were cited earlier, give $\mu=0.573$. It was also indicated earlier that the specific reaction-rate constant for step (2) is the most uncertain of the four rate constants. Table 3 shows some rate data that have been used for reaction (2) by different investigators. None of these rate data have experimental justification for temperatures above $1000 \mathrm{~K}$, and some of the preexponential factors differ by more

\section{TABLE 3}

Various Data for the Rate Constant of Reaction (2) According to the Formula $k_{2}=B_{2} T^{\alpha_{2}} \exp \left(-E_{2} / R^{\circ} T\right)$ $\left[\mathrm{cm}^{3} \mathrm{~mol}^{-1} \mathrm{~s}^{-1}\right]$, with $T$ in [K]

\begin{tabular}{llll}
\hline \multicolumn{1}{c}{$B_{2}$} & $\alpha_{2}$ & $\begin{array}{c}E_{2} / R^{\circ} \\
{[\mathrm{K}]}\end{array}$ & Reference \\
\hline $3.37 \times 10^{13}$ & 0 & 2868 & {$[8]$} \\
$1.20 \times 10^{13}$ & 0 & 2410 & {$[2],[8]$} \\
$5.2 \times 10^{12}$ & 0 & 2088 & {$[8]$} \\
$1.14 \times 10^{13}$ & 0 & 2300 & {$[5],[8]$} \\
$2.3 \times 10^{13}$ & 0 & 2622 & {$[4]$} \\
$2.96 \times 10^{13}$ & 0 & 3000 & {$[1]$} \\
$4.58 \times 10^{6}$ & 2.5 & 3019 & {$[3]$} \\
$7.2 \times 10^{12}$ & 0.5 & 3019 & {$[2]$} \\
$5.4 \times 10^{12}$ & 0 & 2129 & {$[2]$} \\
\hline
\end{tabular}

than an order of magnitude. It was indicated in earlier work [6] that the data of Heimerl and Coffee [5] currently are best justified. Thus, the specific reaction-rate constants selected are $k_{1}=$ $1.896 \times 10^{14} \exp (-11161 / T)\left[\mathrm{cm}^{3} \mathrm{~mol}^{-1} \mathrm{~s}^{-1}\right]$, $k_{2}=1.14 \times 10^{13} \exp (-2300 / T)\left[\mathrm{cm}^{3} \mathrm{~mol}^{-i}\right.$ $\left.\mathrm{s}^{-1}\right], k_{3}=1.38 \times 10^{18} T^{-1} \exp (-171 / T)\left[\mathrm{cm}^{6}\right.$ $\mathrm{mol}^{-2} \mathrm{~s}^{-1}$, and $k_{4}=0.528 \times 10^{13} \exp (+976 /$ $T)\left[\mathrm{cm}^{6} \mathrm{~mol}^{-2} \mathrm{~s}^{-1}\right]$, where $k_{1}, k_{3}$, and $k_{4}$ have been chosen as those rate constants associated with $\mathrm{O}_{2}$ as a third body, because $\mathrm{O}_{2}$ is the dominant species at the hot side of the flame where those reactions are important, and where the units of $T$ are $[K]$. The constant thermal conductivity of the mixture, $\lambda$, and the constant specific heat at constant pressure of the mixture, $c_{p}$, have been chosen as being identical (in a first approximation) with those of $\mathrm{O}_{2}$ at the flame temperature, and the constant Lewis numbers $L_{1}$ and $L_{3}$ also have been evaluated at the flame temperature. The formulas for the evaluation of $\lambda, c_{p}$, and diffusion coefficients have been taken from [5].

From the given rate constants the rate ratios $\kappa_{2 f}$, $\kappa_{3 \mathrm{f}}$, and $\kappa_{4 \mathrm{f}}$ of (13), (14), and (15) can be evaluated as functions of temperature $T_{\mathrm{f}}$. Results of such evaluations have been given earlier [6]. When approximations of zero activation energies for (2), (3), and (4) are introduced, some adjustments of the earlier values occur. A reasonable set of selections is $\kappa_{2 \mathrm{f}}=0.06 e^{E / R^{\circ} T_{\mathrm{f}}}, \kappa_{3 \mathrm{f}}=530(\mathrm{p} /$

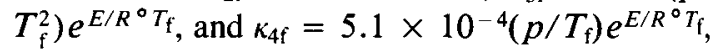
where the units of $p$ and $T_{\mathrm{f}}$ are [atm] and [K], and where $E=22.2 \mathrm{kcal} / \mathrm{mol}$. The resulting values, listed in Table 4, differ from the earlier [6] results usually by less than a factor of 2 , although at the lowest temperatures the present approximate values shown for $\kappa_{2 \mathrm{f}}$ become larger by an order of magnitude than those of [6]. The results demonstrate that at atmospheric pressure $\kappa_{3 \mathrm{f}}$ and $\kappa_{4 \mathrm{f}}$ are sufficiently small to be of importance only in a downstream convective-reactive recombination zone.

With the data given, the temperature and $\mathrm{O}$ atom mass fraction at the downstream end of the primary reaction zone (the upstream end of the recombination zone) can readily be calculated. For this purpose, (21) can be used when the steady state for $\mathrm{O}$ applies, (41) for the two-zone regime 
TABLE 4

Reaction-Rate Ratios of (13), (14), and (15) for the Ozone Decomposition Flame

\begin{tabular}{rlcc}
\hline \multicolumn{1}{l}{$\begin{array}{l}T_{\mathrm{f}} \\
{[\mathrm{K}]}\end{array}$} & \multicolumn{1}{c}{$\begin{array}{c}\boldsymbol{k}^{-1} \kappa_{3 \mathrm{f}} \\
{\left[\mathrm{atm}^{-1}\right]}\end{array}$} & $\begin{array}{c}p^{-1} \kappa_{4 \mathrm{f}} \\
{\left[\mathrm{atm}^{-1}\right]}\end{array}$ \\
\hline 800 & $6.87 \times 10^{4}$ & $9.52 \times 10^{2}$ & $7.29 \times 10^{-1}$ \\
1000 & $4.22 \times 10^{3}$ & $3.74 \times 10^{1}$ & $3.58 \times 10^{-2}$ \\
1500 & $1.02 \times 10^{2}$ & $4.03 \times 10^{-1}$ & $5.78 \times 10^{-4}$ \\
2000 & $1.59 \times 10^{1}$ & $3.53 \times 10^{-2}$ & $6.75 \times 10^{-5}$ \\
2500 & 5.21 & $7.39 \times 10^{-3}$ & $7.77 \times 10^{-5}$ \\
3000 & 2.48 & $2.44 \times 10^{-3}$ & $3.53 \times 10^{-6}$ \\
3500 & 1.46 & $1.05 \times 10^{-3}$ & $2.07 \times 10^{-6}$ \\
4000 & 0.98 & $5.41 \times 10^{-4}$ & \\
\hline
\end{tabular}

or for the limit $K \rightarrow 0$ of the merged regime, and (20) along with the results [6] of the mergedregime analysis (Appendix A) otherwise. Figure 6 shows the results of these calculations, as a function of the initial ozone mole fraction $X_{3 u}$, for an initial temperature of $300 \mathrm{~K}$. These results are independent of pressure so long as the recombination zone retains a convective-reactive character, which it does at least for $p<10$ atm (see below). The Zel'dovich number (16) is readily evaluated from these results; its value is indicated at two

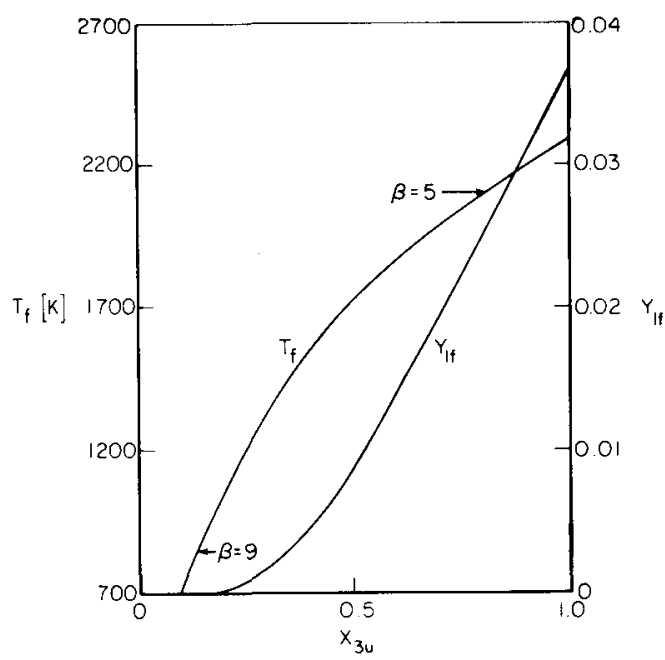

Fig. 6. Flame temperature $T_{f}$ (ahead of the recombination zone) and maximum mass fraction $Y_{1 f}$ of oxygen atoms as functions of the initial ozone mole fraction for the ozone decomposition flame at an initial temperature of $T_{\mathrm{u}}=300 \mathrm{~K}$. points on the $T_{\mathrm{f}}$ curve, from which it is seen that the value decreases with increasing $X_{3 u}$.

When the inequality (44) is applied to the ozone flame with the data given here, it is found that under no conditions whatever can this flame be in the two-zone regime. In this respect, it is interesting that even in their early study, von Kármán and Penner [10] reasoned that the forward diffusion of $\mathrm{O}$ atoms is not significant in this flame. The improved data currently available support this conclusion not only for the particular case considered in [10] but also at all pressures, initial temperatures, and initial ozone mole fractions. The recent findings $[6,7]$ to the effect that a steady state for $\mathrm{O}$ prevails in the primary reaction zone at low values of $X_{3 u}$ while a merged regime occurs at higher values of $X_{3 \mathrm{u}}$ is supported by the present results, which indicate that, furthermore, the merged regime extends all the way to $X_{3 u}=1$.

The burning velocity of the ozone flame at atmospheric pressure and at an initial temperature of $300 \mathrm{~K}$ is shown as a function of $X_{3 u}$ in Fig. 7 . The points are the numerical integrations of [5], selected for comparison here and in [6] since comparisons with experiments and with other numerical integrations were given in [5]. It is appropriate here to treat the results of [5] as the correct results since the input data adopted were those of [5]. The dashed curve in Fig. 7 is the result of the steady-state approximation and the solid curve the most well justified calculation for the merged regime, taken from [6]. The agreement 


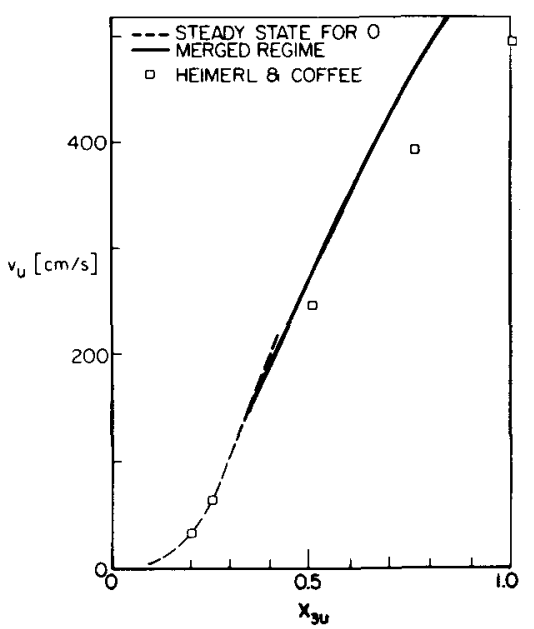

Fig. 7. The burning velocity as a function of the initial ozone mole fraction according to the numerical integrations of [5] (points), the steady state for $O$ (dashed line), and the merged regime (solid line) [6] for atmospheric pressure and an initial temperature of $T_{\mathrm{u}}=300 \mathrm{~K}$.

in the range where the steady state applies is excellent, but as $X_{3 u}$ increases in the merged regime the agreement becomes progressively poorer. The disagreement here is attributable entirely to inaccuracy of a one-term expansion in $\beta^{-1}$. Accuracies better than $10 \%$ or $20 \%$ in $v_{u}$ simply are not to be expected from an asymptotic analysis in which only the first term of the expansion is retained. The differences between the points and the solid curve, even where the difference is the greatest, are within the accuracy estimates of the asymptotic results. The excellent agreement where the steady state applies is purely fortuitous. Carrying the expansion to the next order in $\beta^{-1}$ for the merged regime gives much better agreement. Development of this two-term expansion is straightforward but somewhat tedious and has been performed only recently [11]. A twoterm expansion has been developed for the steadystate portion of the curve [6], and as is to be expected from the agreement in Fig. 7, it gave negligible differences from the one-term result.

Numerical integrations have been performed to find the dependence of the burning velocity on the pressure and the initial temperature [12], but only for $X_{3 u}=0.5$, and with transport descriptions less detailed than in the earlier work [5]. The results for the temperature dependence are shown in Fig. 8 and compared there with the asymptotic results for the merged regime. These results differ from those in Fig. 7 because of the differing transport models. The good agreement in Fig. 8 for $X_{3 u}=$ 0.5 apparently reflects inaccuracies in the numerical results associated with the simplified transport models [12] and inaccuracies in the asymptotic results associated with retaining only the leading order in $\beta^{-1}$; these inaccuracies happen to bring the two results together even at the higher temperatures, where both are likely to be poor. The numerical integrations show essentially no dependence of $v_{\mathrm{u}}$ on pressure for $0.1 \mathrm{~atm} \leq p \leq 10 \mathrm{~atm}$ at $T_{\mathrm{u}}=300 \mathrm{~K}, X_{3 \mathrm{u}}=0.5$ and were not carried beyond this range. This insensitivity is entirely in accord with the present results, e.g., as seen from (A4) and (22), as it must be because of the controlling mechanism involving only steps (1) and (2). At $10 \mathrm{~atm} v_{\mathrm{u}}$ had decreased by about $1 \%$ [12], suggesting the onset of a contribution from (3) or (4).

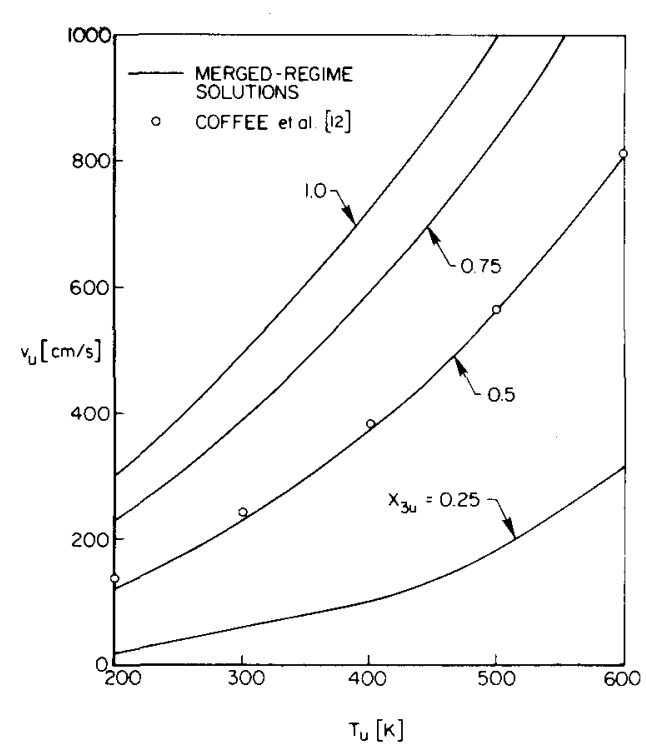

Fig. 8. The burning velocity at atmospheric pressure as a function of the initial temperature for various values of the initial ozone mole fraction, according to the numerical integrations of [12] (points) and the solutions for the merged regime (lines). 


\section{REGIMES FOR THE OZONE FLAME}

From the preceding results it is clear that at $p=1$ atm and $T_{\mathrm{u}}=300 \mathrm{~K}$ the ozone decomposition flame has a primary reactive-diffusive zone in the merged regime for all $X_{3 u}$, with the steady-state approximation for $\mathrm{O}$ being rather good for $X_{3 \mathrm{u}} \leqslant$ 0.4 , and with a convective-reactive recombination zone downstream from the primary reaction zone. In this convective-reactive zone $\mathrm{O}_{3}$ maintains a steady state (25) and steps (3) and (4) both contribute to recombination, although step (3) is most important for $X_{3 u} \geqslant 0.4(J>1)$ and step (4) for $X_{3 \mathrm{u}} \leqslant 0.3(J<1)$. These last estimates are obtained from (24) and the associated discussion, with the rate parameters given at the beginning of the preceding section and with $Y_{1 \mathrm{f}}$ calculated as described in footnote 4.

If $T_{\mathrm{u}}$ is changed at $p=1 \mathrm{~atm}$, then there are changes in the values of $X_{3 u}$ at which the steady state for $\mathrm{O}$ becomes good and at which $J=1$. Figure 9 illustrates these changes. It is seen from the dashed line labeled $J=1$ that as $T_{\mathrm{u}}$ increases

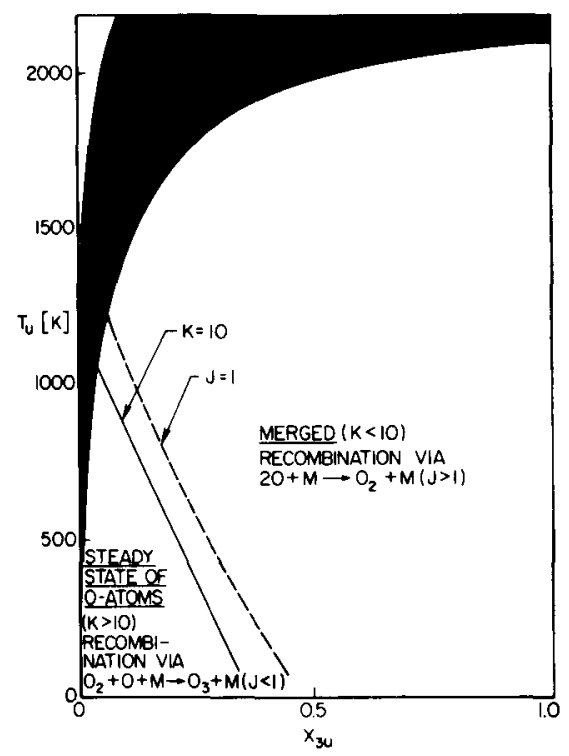

Fig. 9. Criterion for attainment of a steady state for $\mathrm{O}$ atoms, boundary separating different recombination mechanisms, and limit of nonacceleratory flame propagation, as functions of initial ozone mole fraction and initial temperature, for pressures below $10 \mathrm{~atm}$. the range of $X_{3 u}$ over which step (3) dominates the recombination increases, mainly because of the increase in $Y_{\text {If }}$ with increasing $T_{\mathrm{f}}$. The solid line labeled $K=10$ provides a conservative criterion for use of the steady state for O. In Fig. 7 it was found that at $T_{\mathrm{u}}=300 \mathrm{~K}$ this steady state is not too bad even at $K \approx 2$, corresponding to $Y_{3 u}=0.4$. Therefore in Fig. 9 the boundary represented by the solid line may be allowed to move to the right if a somewhat less conservative criterion is adopted. In fact, the dashed line may represent the most reasonable rough location of this boundary. The near-coincidence of the two is a fortuitous consequence of the values of the rate constants, although their slopes always must be essentially the same because they both are governed primarily by the dependence of $Y_{\text {If }}$ on $T_{\mathrm{f}}$.

The shaded region in the upper part of Fig. 9 is a boundary of normal, nonacceleratory flame propagation. The upper edge of this region has been calculated from (43) by setting $T_{\mathrm{f}}=T_{\mathrm{u}}$, and the lower edge has been obtained by putting $\beta=1$ in the two-zone or merged-regime computation (each giving essentially the same result). Although (44) is never satisfied for the ozone flame, it is seen from Fig. 3 that this criterion becomes inaccurate when $\theta_{\mathrm{t}}$ becomes small, underpredicting the burning velocity in the two-zone regime, and formal application of the criterion that the two-zone $v_{\mathrm{u}}$ exceed the merged-regime $v_{\mathrm{u}}$ must lead to a transition to the two-zone regime somewhat within the shaded region. This is not physically significant because of the small Zel'dovich number in the shaded region. Throughout the shaded region(and beyond) flame propagation would be acceleratory, and reactions in the gas ahead of the flame could not be neglected; at temperature $T_{\mathrm{u}}$ the rate of (1) already would be comparable with that of (2), so that the basis of activation-energy asymptotics would fail here for this kinetic mechanism. In fact, noticeably acceleratory propagation is to be anticipated already at $\beta \approx 3$, and a practical limit beyond which a welldefined, steady burning velocity no longer exists therefore would lie below the shaded region, roughly at $T_{\mathrm{u}} \approx 1200 \mathrm{~K}$ for all $X_{3 \mathrm{u}}$ above a flammability limit. For the ozone flame, this failure of the concept of a burning velocity is a 
reality and not a consequence of kinetic approximations.

These observations complete the map of regimes for $p=1$ atm. Since the recombination zone is convective-reactive at $p=1$ atm and remains so as $p$ is reduced further, and since both of the recombination steps have $\kappa_{\mathrm{i}} \sim p$, there is no change whatever in Fig. 9 as pressure is decreased. Reducing the pressure merely lengthens the recombination zone. It is readily found from (25) and the expression following it that the length of the recombination zone (measured in units of the preheat-zone thickness) is of order $\beta^{-2}\left(\kappa_{3} / 2 \kappa_{2}\right.$ $\left.+2 \kappa_{4}\right)^{-1}$ and that $Y_{1}$ and $Y_{3}$ there are of order $\left(2 \kappa_{2}\right)^{-1}$ and $\kappa_{4} / \kappa_{2}$, respectively: Thus, $Y_{1}$ is fixed, $Y_{3}$ is proportional to $p$, and the relative length is inversely proportional to $p$. If $p$ is increased, then the length decreases until eventually the recombination zone begins to affect the burning velocity. If $\kappa_{3} / 2 \kappa_{2}+2 \kappa_{4}$ is taken to be of order $\beta^{-m}$, then for $m>2$ the recombination zone remains convective-reactive, at $m=2$ it becomes convective-reactive-diffusive and begins to have an influence on $v_{u}$, for $0<m<2$ it is reactive-diffusive (and influences $v_{\mathrm{u}}$ ) but still thicker than the primary reaction zone, and at $m=0$ it merges with the primary reaction zone, so that the reaction goes to completion in this reaction zone $\left(\theta_{\mathrm{f}}=1\right)$. The rate data enable us to estimate $m$ for different pressures.

Table 5 summarizes the dependence of relevant combination of recombination-rate parameters on $T_{\mathrm{f}}$. For sufficiently high flame temperatures this result leads to $m \geq 3$ at $1 \mathrm{~atm}, m \geq 2$ at $10 \mathrm{~atm}$, and $m \geq 1$ at $100 \mathrm{~atm}$; at lower flame temperatures $m$ is smaller. The most distinctive bounda-

\section{TABLE 5}

Dependence of the Orders of Magnitude of Recombination-Rate Ratios on Flame Temperature for the Ozone Flame

\begin{tabular}{lcccc}
$T_{\mathrm{f}}[\mathrm{K}]$ & $\sim 800$ & $\sim 950$ & -1200 & $\$ 2200$ \\
\hline $\begin{array}{l}p^{-1}\left(\kappa_{3} / 2 \kappa_{2}+2 \kappa_{4}\right) \\
{\left[\mathrm{atm}^{-1}\right]}\end{array}$ & 1 & $10^{-1}$ & $10^{-2}$ & $10^{-3}$
\end{tabular}

ries of regimes are the conditions under which the recombination zone assumes a convective-reactive-diffusive character $(m=2)$ and those under which it becomes indistinguishable from the primary reaction zone $(m=0)$. Estimates for approximate locations of these boundaries are illustrated in a plane of $X_{3 \mathrm{u}}$ and $T_{\mathrm{u}}$ in Fig. 10 and in a plane of $T_{\mathrm{u}}$ and $p$ in Fig. 11. The solid lines in these two figures correspond to $m=2$; the dashed lines in Fig. 10 show the coalescence condition, $m$ $=0$. These curves were generated by first selecting specific values for $m, p$, and $X_{3 u}$, then adjusting $T_{\mathrm{u}}$ to satisfy the formula $\kappa_{3} / 2 \kappa_{2}+2 \kappa_{4}=$ $\beta^{-m}$. For the evaluation of the $\kappa_{i}$ and $\beta, T_{\mathrm{f}}$ was found by use of (20) with $Y_{1 \mathrm{f}} \approx 0$ and with $c_{\mathrm{p}}$ that of $\mathrm{O}_{2}$ at temperature $T_{\mathrm{f}}$. By definition, the curves for $m=0$ and for $m=2$ meet at the boundary where $\beta=1$ in Fig. $10 ; \beta$ increases with increasing $X_{3 \mathrm{u}}$ along each of these curves and reaches a peak value of about 10 at low initial temperatures. In Fig. $10 v_{\mathrm{u}}$ is affected by the recombination steps (or, for the dashed lines, there is a single reaction zone with $\theta_{\mathrm{f}}=1$ ) at points below the lines. It is thus seen from Fig. 11, for example, that $p \gtrsim 100 \mathrm{~atm}$ is needed for the recombination steps to be important at $X_{3 u}=1$ for $T_{\mathrm{u}}=300 \mathrm{~K}$.

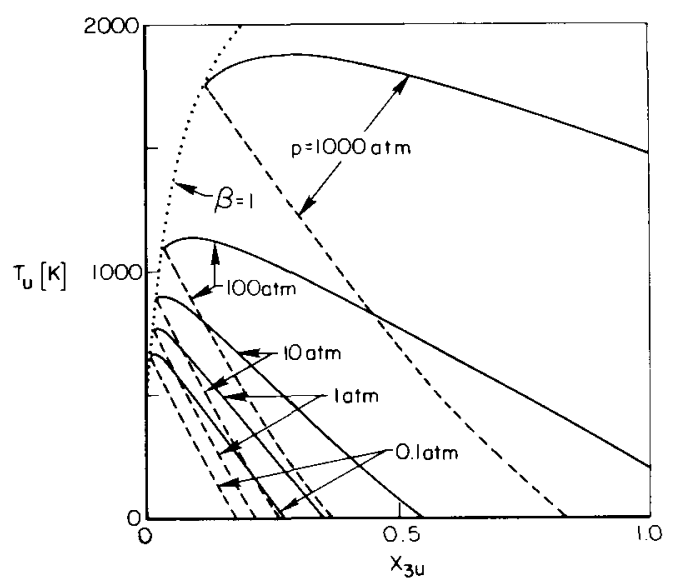

Fig. 10. Boundaries along which the recombination zone begins to influence the burning velocity (solid lines) and merges with the primary reaction zone (dashed lines) in a plane of initial ozone mole fraction and initial temperature, for a variety of pressures. 


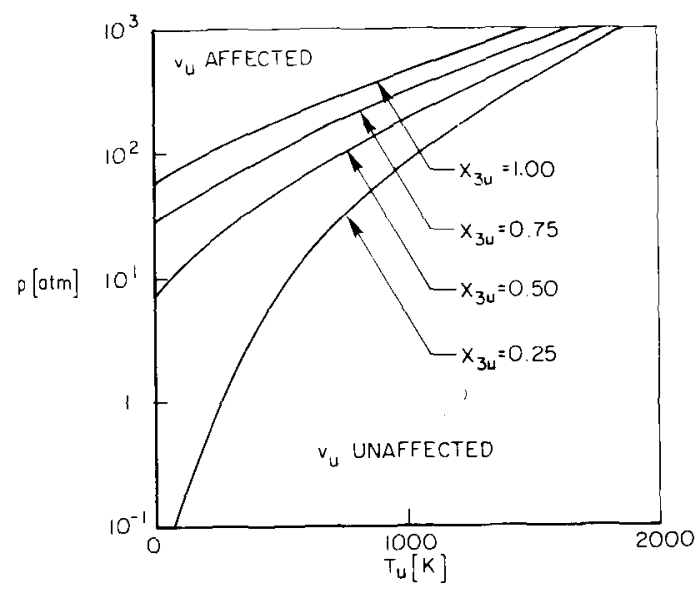

Fig. 11. Boundaries along which the recombination zone begins to influence the burning velocity in a plane of initial temperature and pressure for various initial ozone mole fractions.

\section{CONCLUDING REMARKS}

The greatest uncertainties in the results derived herein for the ozone decomposition flame occur at elevated flame temperatures and arise from uncertainties in the rate constants for steps (1) and especially (2), as emphasized in Table 3. Improved rate data for $\mathrm{O}+\mathrm{O}_{3} \rightarrow 2 \mathrm{O}_{2}$ at high temperatures could eliminate uncertainties that sometimes reach as much as an order of magnitude in $v_{\mathrm{u}}$ and could improve confidence in the prediction that the two-zone structure does not occur for nonacceleratory propagation of the ozone decomposition flame.

The excellent agreement, found earlier [6] for burning velocities of the ozone decomposition flame, between results of a one-term expansion in $\beta^{-1}$ and those of numerical integrations of the full equations, for conditions corresponding to a steady state for $\mathrm{O}$ atoms, was fortuitous in that it is not a general property of results of activationenergy asymptotics. In the merged regime (e.g., at higher $X_{3 u}$ ) the expected errors of 10-20\% occur. To reduce these errors to a few percent it is necessary to extend the merged-regime analysis to a two-term expansion in $\beta^{-1}$ [11].

The asymptotic approach provides explicit criteria distinguishing different regimes of flame prop- agation and in some regimes gives prescriptions for calculating burning velocities from simple formulas. In other regimes, parametric results obtained by numerical integrations of two-point boundary-value problems for ordinary differential equations give the prescriptions for calculating burning velocities from activation-energy asymptotics. The approach is well suited to identifying scaling laws and general characteristics of flame structures.

Kinetic mechanisms of the ozone-flame type lead to a wider variety of types of flame structures than might be expected. In addition to structures involving a steady state for the reaction intermediary in a thin reaction zone, there are structures with a thin reaction zone in which the steady state is not achieved (the merged regime) and structures in which the intermediary is produced in a thicker, hot reaction zone (the generation zone) and diffuses into the preheat zone to react exothermically there (transforming it into an exothermic zone), as in the two-zone regime identified and analyzed herein. Another attribute is the recombination zone, which under suitable conditions coalesces with the primary reaction zone and which exhibits regimes involving direct recombination or recombination through regeneration of the reactant which maintains a steady state. Identification of these different types of structures is facilitated by asymptotic methods.

\section{APPENDIX A: ANALYSIS OF THE MERGED REGIME}

In the merged regime the preheat-zone solutions to (5), (6), and (7) are $\theta=\theta_{\mathrm{f}} e^{x}, Z=0$, and $Y=1$ - $e^{L_{3} x}$ in the first approximation, where the reaction zone has been placed at $x=0$. Appropriately stretched variables for the reaction zone are $\eta$ $=x \sqrt{\Lambda L_{3} / 2}, \phi=\beta\left(\theta_{\mathrm{f}}-\theta\right) / \theta_{\mathrm{f}}, y=\beta Y / L_{3}$, and $z$ $=\beta Z / L_{1}$. In terms of these variables, with $\kappa_{3}$ and $\kappa_{4}$ small enough to be negligible in the reaction zone and to provide zero-gradient matching conditions downstream, (5) and (7) give, in the first approximation,

$$
d^{2} y / d \eta^{2}=K y z e^{-b \phi}+y e^{-\phi}
$$


and

$$
d^{2} \phi / d \eta^{2}=(1+\mu) K y z e^{-b \phi} / \mu-(1-\mu) y e^{-\phi / \mu}
$$

where $K$ is defined in (22). Equation (18) demonstrates that to achieve matching the order of magnitude of $1-\theta_{\mathrm{f}}$ cannot be greater than $\beta^{-1}$, and that moreover (from integration and matching)

$z=z_{\mathrm{f}}+\phi-y$,

where $z_{\mathrm{f}}$ is the constant value that $z$ approaches as $\eta \rightarrow \infty$. Here the factor $e^{-b \phi}$ has been inserted in (A1) and (A2) to exhibit the modification that arises if the activation energy of step (2) is not zero. Matching conditions for (A1) and (A2) are that $y$ and $\phi$ approach zero as $\eta \rightarrow \infty$ and that $d y /$ $d \eta$ and $d \phi / d \eta$ approach the same constant value $-V$ as $\eta \rightarrow-\infty$, where a nondimensional burning velocity is

$$
V \equiv \sqrt{2 \beta^{2} / \Lambda L_{3}}=\beta v_{\mathrm{f}} / \sqrt{L_{3 \mathrm{f}} \lambda_{\mathrm{f}} k_{\mathrm{lf}} / 2 W c_{\mathrm{pf}}},
$$

in which use has been made of (11). From (A3) it then follows that $d z / d \eta \rightarrow 0$, so that $z$ approaches a constant, say $z=z_{i}$, as $\eta \rightarrow-\infty$, the value of which is seen from (A1) or (A2) necessarily to be zero if $b=0$. With $\mu, K$, and $b$ as parameters of order unity (as well as for $b=0$ ), the problem posed here serves to determine unique solutions and values of $V$ and $z_{\mathrm{f}}$.

In general, the orderings are to be modified if $\mu$ or $K$ approaches limiting values. As $\mu \rightarrow 1$ with $K$ of order unity there is no change, but as $\mu \rightarrow 0$ with $K$ of order unity, it is found that $y$ becomes of order $\mu$, so that working with $\phi$ and $z$, eliminating $y$ by (A3), effects a simplification. Of greater interest here are the limits of large and small values of $K$, with $\mu$ of order unity. For large values of $K$ it has been shown [6] that appropriate expansions are $y=y_{0}+K^{-1} y_{1}+\cdots, \phi=\phi_{0}$ $+K^{-1} \phi_{1}+\cdots, z=K^{-1} z_{0}+K^{-2} z_{1}+\cdots$, and $V=V_{0}+K^{-1} V_{1}+\cdots$, and the solutions give

$$
V=2+\left\{1-[1-\mu(1-b)] /(2-b)^{2}\right\} / K+\cdots
$$

and

$z_{\mathrm{f}}=1 / K-2 \mu(1-b) / K^{2}+\cdots$

(for $0 \leq b \leq 1$ ), as well as $z_{i}$ small compared with $K^{-2}$, thereby demonstrating a smooth approach to the steady state for $O$. Investigation of the limit of small values of $K$ entails further effort, as outlined below for $b=0$.

As $K$ becomes small the variables $\hat{y} \equiv K y$ and $\hat{z}$ $\equiv K z$ tend to remain of order unity, so that it is more convenient to write (A1), (A2), and (A3) with $b=0$ as

$d^{2} \hat{y} / d \eta^{2}=\hat{y} \hat{z}+\hat{y} e^{-\phi}$,

$K d^{2} \phi / d \eta^{2}=\hat{y} \hat{z}(1+\mu) / \mu-\hat{y} e^{-\phi}(1-\mu) / \mu$,

and

$\hat{z}=\hat{z}_{f}-\hat{y}+K \phi$.

The conservation equation for $\hat{z}$, obtained from these and corresponding to $(6)$, is

$\mu d^{2} \hat{z} / d \eta^{2}=\hat{y} \hat{z}-\hat{y} e^{-\phi}$.

Boundary conditions for $\hat{y}$ and $\hat{z}$ are $\hat{y}(\infty)=0$, $\hat{z}(\infty)=\hat{z}_{f}=$ constant, $\hat{z}(-\infty)=0$, and $d \hat{y} / d \eta$ $\rightarrow-\hat{V}$ as $\eta \rightarrow-\infty$, where $\hat{V} \equiv K V$. As $K \rightarrow 0$, a transition layer develops, of thickness of order $\sqrt{K}$, and it is convenient to employ the translational invariance of the problem as defined here to place this layer at $\eta=0$. With this convention, from (A8) and (A9) the solutions

$\hat{y}=\hat{z}_{\mathrm{f}}-\hat{z}$

and

$\hat{z}=e^{-\phi}(1-\mu) /(1+\mu)$

are obtained for $\eta>0$, the last of which identifies the steady-state approximation for temperature in these variables and also implies that

$\hat{z}_{\mathrm{f}}=(1-\mu) /(1+\mu)$.

Use of (A11) and (A12) in (A7) yields an equation for $\hat{y}$ that can be integrated once to give

$$
d \hat{y} / d \eta=-2 \hat{y} \sqrt{\hat{z}_{\mathrm{f}} / 2-\hat{y} / 3} / \sqrt{1-\mu},
$$


another integral of which would complete the solution in the first approximation for $\eta>0$.

For $\eta<0, \phi$ will be large enough for step (1) to be frozen, so that $\hat{\phi}=K \phi$ is the appropriate rescaled temperature variable of order unity. Substitution of this rescaled variable into (A8), (A9), and (A10) yields, in the first approximation for small $K$,

$\mu d^{2} \hat{\phi} / d \eta^{2}=(1+\mu) \hat{y} \hat{z}$,

$\mu d^{2} \hat{z} / d \eta^{2}=\hat{y} \hat{z}$

and

$\hat{y}=\hat{z}_{\mathrm{f}}-\hat{z}+\hat{\phi}$.

A linear combination of (A15) and (A16) is readily integrated twice with application of the matching conditions for $\eta \rightarrow-\infty$ to give

$\hat{\phi}=(1+\mu) \hat{z}-\hat{V} \eta+\mu \hat{c}-\hat{z}_{\mathrm{f}}$,

where a constant of integration has been written for convenience as $\mu \hat{c}-\hat{z}_{\mathrm{f}}$ with $\hat{c}$ arbitrary. Use of (A17) and (A18) in (A16) gives the differential equation

$d^{2} \hat{z} / d \eta^{2}=\hat{z}[\hat{z}-(\hat{V} / \mu) \eta+\hat{c}]$,

which is to be integrated subject to $\hat{z}(-\infty)=0$ and to suitable matching conditions at $\eta=0$ to complete the first approximation to the solution for $\eta<0$.

Development of the matching conditions entails considering the transition layer at $\eta=0$. An appropriate independent variable for this layer is $\zeta$ $=\eta / \sqrt{K}$, and expansions of $\hat{y}, \hat{z}$, and $\phi$ in powers of $\sqrt{K}$ arise. In the first approximation it is seen from (A7) and (A10) that $\hat{y}$ and $\hat{z}$ must be linear in the stretched variable, and therefore from matching it is found that continuity of these functions and of their first derivatives across the transition layer may be applied to the outer solutions. With values and slopes at $\eta=0$ identified by subscript 0 , it is then seen from (A11) that

$\hat{z}_{0}=\hat{z}_{\mathrm{f}}-\hat{y}_{0}$

and

$(d \hat{z} / d \eta)_{0}=-(d \hat{y} / d \eta)_{0}$ may be employed as boundary conditions for (A19), with $(d \hat{y} / d \eta)_{0}$ related to $\hat{y}_{0}$ by (A14). The expansion of (A8) in the stretched variable in the first approximation becomes

$d^{2} \phi / d \zeta^{2}=\hat{y}_{0} \hat{z}_{0}(1+\mu) / \mu-e^{-\phi} \hat{y}_{0}(1-\mu) / \mu$

From (A12) it is seen that

$\phi_{0}=\ln \left\{(1-\mu) /\left[(1+\mu) \hat{z}_{0}\right]\right\}$

is the value that $\phi$ must approach as $\zeta \rightarrow \infty$ for matching. The matching condition for $\phi$ as $\zeta \rightarrow$ $-\infty$ may be developed from (A18), which when evaluated at $\eta=0$ first yields

$\hat{c}=\left[\hat{z}_{\mathrm{f}}-(1+\mu) \hat{z}_{0}\right] / \mu$

to prevent $\hat{\phi}$ from being too large for matching. The next two terms in the expansion of (A18) about $\eta=0$ yield, respectively,

$\hat{V}=(1+\mu)(d \hat{z} / d \eta)_{0}$

and

$\phi=(1+\mu)\left(d^{2} \hat{z} / d \eta^{2}\right)_{0} \zeta^{2} / 2$

as matching requirements, the last of which is to be applied to (A22) as $\zeta \rightarrow-\infty$.

Use of (A19) and (A24) in (A26) reduces the matching condition to $\phi=\hat{\zeta}^{2} / 2$, where

$\hat{\zeta} \equiv\left[\hat{z}_{0}\left(\hat{z}_{\mathrm{f}}-\hat{z}_{0}\right)(1+\mu) / \mu\right]^{1 / 2} \zeta$.

Use of (A20) and (A27) in (A22) shows that the differential equation can be written as

$d^{2} \phi / d \xi^{2}=1-e^{-\left(\phi-\phi_{0}\right)}$,

where $\phi_{0}$ is given by (A23). The inverse solution satisfying the boundary conditions can then be written as $\hat{\xi}=-\int_{\hat{a}}^{\phi}(2 \hat{g})^{-1 / 2} d \phi$, where

$\hat{g} \equiv\left(\phi-\phi_{0}\right)-\left[1-e^{-\left(\phi-\phi_{0}\right)}\right]$,

and the relationship

$2 \sqrt{\hat{a}}=\int_{\hat{a}}^{\infty}\left(\hat{g}^{-1 / 2}-\phi^{-1 / 2}\right) d \phi$

determines $\hat{a}$ as a function of $\phi_{0}$. A representative transition-layer solution for $\phi(\hat{\zeta})$ is shown in Fig. 


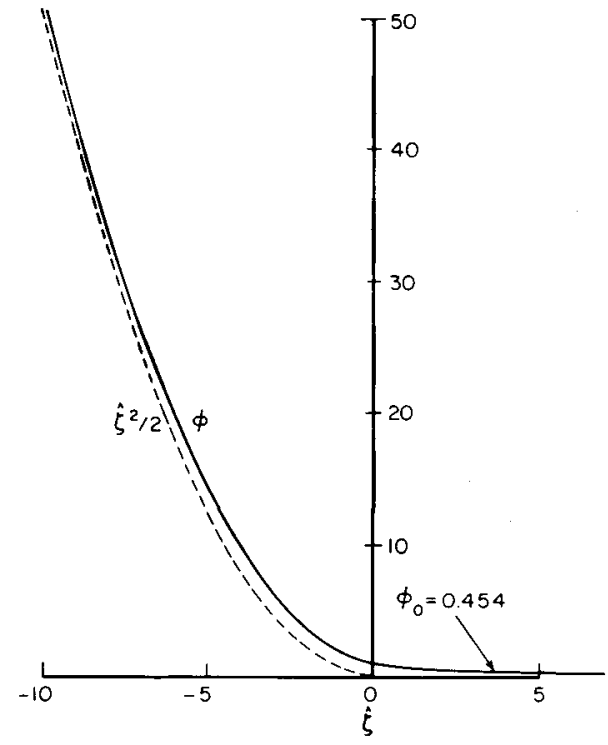

Fig. 12. Transition-layer solution for $\phi_{0}=0.454$.

12, where the parameter selected corresponds to the ozone flame.

Finding $\hat{V}$ entails calculating a numerical solution to (A19). With the new variable $\hat{\eta} \equiv\left[\hat{z}_{\mathrm{f}}-(1\right.$ $\left.+\mu) \hat{z}_{0}-\hat{V} \eta\right] / \mu$, in view of (A24) the differential equation becomes

$d^{2} \hat{z} / d \hat{\eta}^{2}=(\mu / \hat{V})^{2} \hat{z}(\hat{z}+\hat{\eta})$,

which is to be solved subject to $\hat{z}=0$ at $\hat{\eta}=\infty$ and to appropriate boundary conditions at $\hat{\eta}=\left[\hat{z}_{\mathrm{f}}\right.$ $\left.-(1+\mu) \hat{z}_{0}\right] / \mu$, viz., $\hat{z}=\hat{z}_{0}$ and, from (A25), $d \hat{z} / d \hat{\eta}=-\mu /(1+\mu)$. Use of (A20) and (A21) in (A14) provides an expression for $(d \hat{z} / d \eta)_{0}$ in terms of $z_{0}$, and this result may be used in (A25) to show that

$\hat{V}=\sqrt{2 / 3}\left(\hat{z}_{\mathrm{f}}-\hat{z}_{0}\right) \sqrt{\hat{z}_{\mathrm{f}}+2 \hat{z}_{0}}(1+\mu) / \sqrt{1-\mu}$.

Substitution of (A30) into (A29) results, in view of (A13), in a problem in which only $\mu$ and $\hat{z}_{0}$ appear as constants. Since the problem is that of a secondorder differential equation with three boundary conditions, its solution determines $\hat{z}_{0}$ as a function of $\mu$. Use of this result and of (A13) in (A30) then gives $\hat{V}$ as a function of $\mu$. Results of the numerical integration are presented graphically in Fig. 13, and a few selected values of $\hat{V}$ are listed in

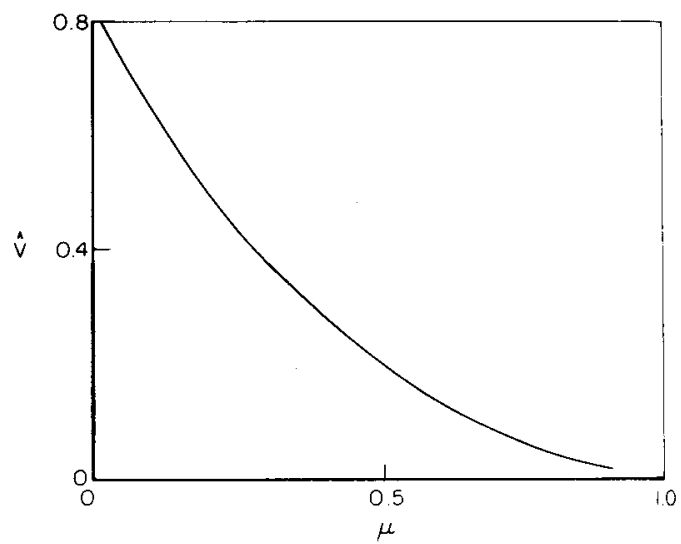

Fig. 13. Nondimensional burning velocity $\hat{V}=K V$ for the merged regime in the limit $K \rightarrow 0$, obtained from (A29) and (A30) by numerical integration.

TABLE 6

Nondimensional Burning Velocity $\hat{V}$ of the Merged Regime for $K \rightarrow 0$

\begin{tabular}{llllll}
\hline$\mu$ & 0.1 & 0.3 & 0.5 & 0.7 & 0.9 \\
$\hat{V}$ & 0.636 & 0.376 & 0.201 & 0.085 & 0.015
\end{tabular}

Table 6. For example, at $\mu=0.573$ it is found that $\hat{V}=0.153$ and, from (A13), $\hat{z}_{\mathrm{f}}=0.271$ for this $\mu$. Thus, it is found that for small $K$

$V=0.153 / K+\cdots, \quad z_{\mathrm{f}}=0.271 / K+\cdots$

for $\mu=0.573$.

This work was supported by the National Science Foundation through Grant \#CPE 8215391. The German Academic Exchange Service (DAAD) provided $B$. $R$. with a scholarship of the NATO Committee of Sciences to support his participation in this work. 